\title{
Chapter
}

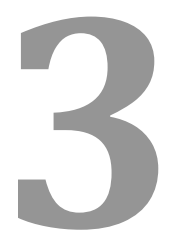

\section{BIODEGRADABLE POLYMERIC NANOMATERIALS}

Géraldine Rohman ${ }^{1 *}$ and Jolanda Spadavecchia ${ }^{2}$

${ }^{1}$ Université Paris 13, Sorbonne Paris Cité, Laboratoire CSPBAT, UMR CNRS 7244, Institut Galilée, 99 avenue JB Clément, 93430 Villetaneuse, France

${ }^{2}$ Université Paris 13, Sorbonne Paris Cité, Laboratoire CSPBAT, UMR CNRS 7244, UFR SMBH, 74 rue Marcel Cachin, 93017 Bobigny, France 


\section{Contents}

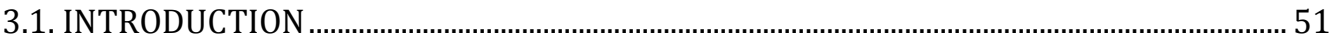

3.2. BIODEGRADABLE POLYMERS........................................................................................... 52

3.2.1. Aliphatic polyesters .............................................................................................5

3.2.2. Other synthetic biodegradable polymers................................................................55

3.2.3. Polysaccharides .....................................................................................................5

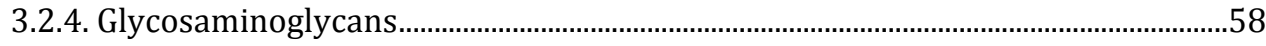

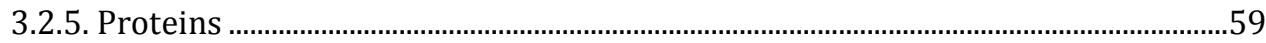

3.3. POLYMERIC NANOFIBRES AND NANOFIBROUS SCAFFOLDS ……………………………... 60

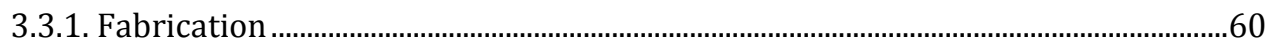

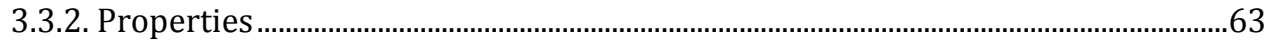

3.3.3. Applications of nanofibrous materials ....................................................................68

3.4. POLYMERIC NANOPARTICLES........................................................................................ 70

3.4.1. Advantages and applications of polymeric nanoparticles ........................................71

3.4.2. Methods for nanoparticle preparation .........................................................................72

3.4.2.1. Self-assembly ............................................................................................

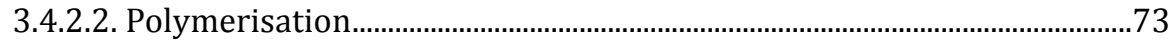

3.4.2.3. Emulsification / solvent evaporation ........................................................73

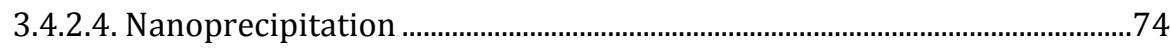

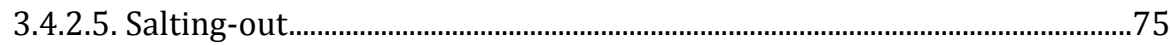

3.4.3. Examples of nanoparticles obtained from biodegradable synthetic and natural polymers ……………………………………………………………………….....77

3.5. SURFACE MODIFICATION ................................................................................................... 79

3.5.1. Surface modification of nanofibrous scaffolds ............................................................8

3.5.2. Coupling strategies for the biofunctionalisation of nanoparticles..........................82

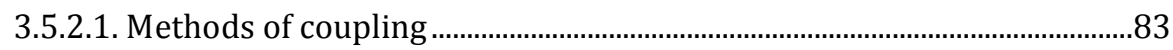

3.5.2.2. Polymers as conjugating agents on PNPs ..................................................84

3.6. CONCLUSION …………………………………………………………………………………….... 86

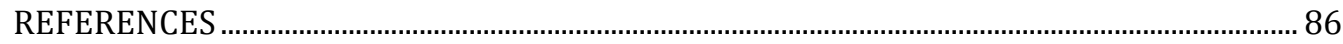




\subsection{INTRODUCTION}

Polymeric nanomaterials are materials ranging from 1-1000 $\mathrm{nm}$ in size in at least one dimension and are associated with having a larger surface area than their larger-scale material cousins. Their peculiar nanosized structure confers different properties to the nanomaterials in comparison to bulk polymers. The main advantages of polymeric nanomaterials are that they can be designed with a variety of compositions, sizes and possible morphologies, surface chemistries, and surface topographies [1]. In recent years, polymeric nanomaterials have attracted increased attention as they can be developed to exhibit a variety of unique properties for a wide range of applications to meet market needs. Indeed, if recent trends continue, polymeric nanomaterials will emerge as the fastest growing domain in the near future [2].

Biodegradable polymers contain functional groups that may be cleaved in vivo leading to the fragmentation of the polymeric chains and therefore to their solubilisation. Biodegradable polymeric materials are used when it is necessary for a medical device to degrade over the time and these materials must fulfil some prerequisites to be considered useful for such purposes: do not impart inflammatory or toxic responses upon implantation, have an acceptable shelf life, possess a degradation rate in physiological conditions in accordance with the intended application, have appropriate initial mechanical properties with pertinent variations over the material degradation period, generate non-toxic degradation products that may be metabolised and cleared from the body, and have appropriate processability and sterisability for the intended application [3].

Biodegradable nanomaterials can be divided into four categories: spheres, hydrogels, micelles and fibres. They have been employed for a wide variety of applications in the biomedical field such as drug or biomolecular delivery systems, vaccines, scaffolds for tissue engineering and even wound dressings $[4,5]$. As biodegradable nanoparticles are digested internally and cleared from the body, they are often chosen over their non-biodegradable counterparts. Moreover, their application for the delivery of drugs and bioactive molecules has been shown to improve the bioavailability, the solubility and the retention time of the encapsulated systems. Indeed, with non-biodegradable drug carriers, the release of therapeutic agents may be poor due to their high molecular weights which can impede diffusion through the polymer matrix. Furthermore, when using biodegradable systems, the interaction with the biological environment is also increased leading to better tissue absorption and intracellular penetration. Therefore, the comparative therapeutic index is increased with enhanced tolerability and low toxicity risks [6]. In tissue engineering applications, scaffolds based on biodegradable nanofibres offer several advantages, such as a large surface area-to-volume ratio, high and 
tunable porosity, malleability to facilitate a wide variety of shapes, superior mechanical properties compared to regular fibres, and finally high axial strength combined with high flexibility. Moreover, their morphologies closely match the architecture of natural tissue extracellular matrix, since nanofibres possess the same dimensions as collagen or cellular cytoskeleton fibres. Therefore, nanofibre-based scaffolds enhance biological activity resulting in better cell attachment, proliferation and maintenance of phenotypic expression [7].

The first section of this chapter presents a background of the biodegradable polymers used in medical applications. Then, the development of nanofibres and nanofibrous scaffolds as well as nanoparticles, with a view to medical applications, is explored. Finally, the surface modifications applied to such nanomaterials are covered in more detail.

\subsection{BIODEGRADABLE POLYMERS}

Biodegradable polymers are divided into two categories. Synthetic degradable polymers have the advantage of not presenting immunogenicity, being synthesised with reliable sources, and being manufactured to obtain predictable properties. They mostly degrade hydrolytically since they contain hydrolytically labile chemical bonds in their backbone. Their degradation rates are influenced by the polymer molecular weight, morphology, crystallinity, as well as the device size and shape [8]. Natural polymers share similar traits with extracellular matrix polysaccharides or proteins. They can be recognised by the biological environment, may avoid toxicity issues, and may aid in the attachment, proliferation and differentiation of cells since they contain biofunctional molecules. However, natural polymers are complex and are difficult to purify and characterise, which in-turn makes them difficult to obtain as uniform raw materials. Moreover, natural polymers are enzymatically degraded and their degradation rates, ranging from a few hours to 6 months, are not easily controlled since enzymatic activity can differ between hosts [9]. The chemical structures of some biodegradable polymers, both natural and synthetic, that are widely used to develop nanomaterials are given in Figure 1. 


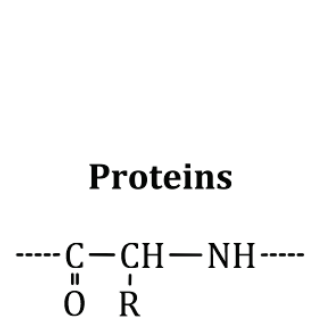

Collagen (Gly-Pro-X)

Gelatin ( $\mathrm{X}=$ others amino-acids)

Silk (Gly-Ser-Gly-Ala-Gly-Ala)

\section{Glycosaminoglycans}

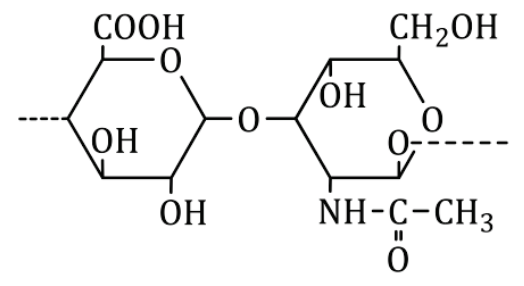

Hyaluronic acid

\section{Polysaccharides}

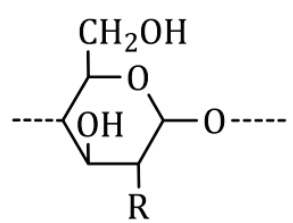

Chitin ( $\left.\mathrm{R}=\mathrm{NHCOCH}_{3}\right)$

Chitosan $\left(\mathrm{R}=\mathrm{NH}_{2}\right)$

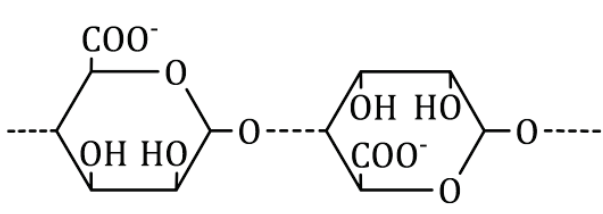

Alginate

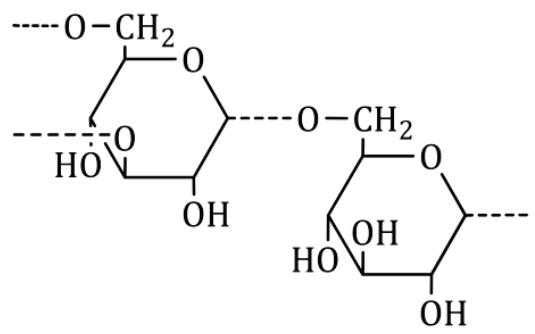

Dextran

Aliphatic polyesters

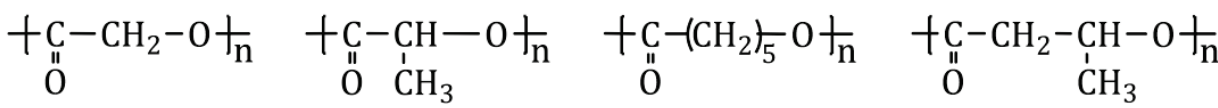

$$
\begin{aligned}
& \begin{array}{llll}
\text { PGA } & \text { PLA } & \text { PCL } & \text { PHB }
\end{array}
\end{aligned}
$$

\section{others}

$$
\underset{0}{\mathrm{C}}-\mathrm{R}-\mathrm{C}_{0}^{\mathrm{C}}-\mathrm{O} t_{\mathrm{n}}
$$

Polyanhydrides

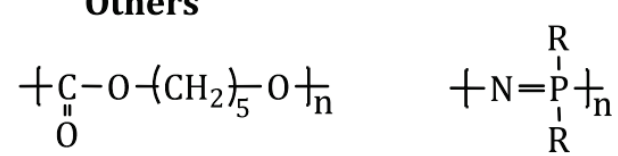

PTMC
Polyphosphazenes

Figure 1. Chemical structures of synthetic and natural biodegradable polymers that are commonly used to develop nanomaterials 


\subsubsection{Aliphatic polyesters}

Aliphatic polyesters, such as polyglycolide (PGA), polylactide (PLA), their copolymers poly(D,L-lactide-co-glycolide) (PLGA), and poly( $\varepsilon$-caprolactone) (PCL), etc., have been widely used for biomedical applications due to their diversity, synthetic versatility, and ease of degradation [3]. They are generally synthesised at high temperatures by ring-opening polymerisation of the corresponding cyclic monomers. They degrade by hydrolysis of the ester linkages along the backbone producing biodegradable metabolite monomers that can be resorbed via metabolic pathways. Their degradation kinetics depend on various factors such as the polymer nature, the monomeric composition, molecular weight, conformation, and physicochemical characteristics [10].

PGA is a highly crystalline polymer that exhibits high melting and glass transition temperatures $\left(T_{\mathrm{m}}=225-233^{\circ} \mathrm{C}, T_{\mathrm{g}}=35-45^{\circ} \mathrm{C}\right)$. It degrades fairly rapidly in vivo (1-12 months) and its degradation results in the production of glycolic acid that, at high concentrations, lowers the $\mathrm{pH}$ of the surrounding tissue and may cause inflammation [9]. PLA is a relatively hard semi-crystalline polymer poly(L-lactide) (PLLA) with a high melting temperature $\left(T_{\mathrm{m}}=170-200^{\circ} \mathrm{C}, T_{\mathrm{g}}=55-65^{\circ} \mathrm{C}\right)$ when synthesised with L-lactide, or an amorphous polymer poly(D,L-lactide) (PDLLA) when obtained with D,L-lactide. PDLLA has only a glass transition temperature of around $55^{\circ} \mathrm{C}$ and therefore has a lower tensile strength in comparison to PLLA. Moreover, PLLA degrades very slowly ( 5 months -5 years), while PDLLA has an intermediate degradation rate (12-16 months) [11]. Consequently, PLGA made of various compositions of D,L-lactide and glycolide monomers, is one of the most successfully used biodegradable polymers in the development of nanomaterials [6]. Indeed, the mechanical properties and the degradation times can be modulated with the lactide/glycolide ratio. Moreover, PLGA degrades into lactic acid and glycolic acid that are metabolised by the body through the Krebs cycle. As a consequence, the use of PLGA nanosystems is associated with minimal systemic toxicity [12]. The US Food and Drug Administration (USFDA) have approved the use of PLGA for human use and nanomedicines. PLGA is soluble in a wide range of solvents and various therapeutic agents have been successfully encapsulated into or adsorbed onto PLGA nanoparticles [6].

PCL is a semi-crystalline polyester with a low glass transition temperature that makes it semi-rigid at room temperature $\left(T_{\mathrm{m}}=58-65^{\circ} \mathrm{C}, T_{\mathrm{g}}=-65\right.$ to $\left.-60^{\circ} \mathrm{C}\right)$. Therefore, PCL may be processed to obtain various shapes, such as spheres, fibres, porous materials, etc., as PCL shows good organic solvent solubility and an innate malleability. Moreover, PCL may be compatibly blended with a wide range of other polymers and is also used as a soft block in polyurethane formulations [13]. PCL degrades slowly (2-3 years) in comparison with the other polyesters and is especially interesting for the preparation of long-term drug delivery systems, such as one-year implantable contraceptives [14]. PCL 
degrades by hydrolysis leading to low-concentrations of caproic acid that does not cause significant negative reactions in the surrounding tissues, and is completely metabolised since caproic acid enters the citric acid cycle. PCL also degrades through enzymatic attack. The bioactivity of PCL is well documented and it is approved by the USFDA for the formulation of nanoparticles $[15,16]$. Various molecules have been successfully incorporated into PCL nanoparticles/nanofibres to increase their therapeutic value [6]. PCL-based nanofibres have also widely been developed as scaffolds for tissue engineering $[16,17]$.

Poly(3-hydroxybutyrate) (PHB) is the simplest member of the poly(3-hydroxyalkanoate) family. This natural polyester can be produced biotechnologically leading to a high semi-crystalline polymer $\left(T_{\mathrm{m}}=168\right.$ $182^{\circ} \mathrm{C}, T_{\mathrm{g}}=1-15^{\circ} \mathrm{C}$ ). PHB could be used for various applications in the biomedical field due to its biocompatibility and biodegradability, as well as the non-cytotoxicity of its metabolic products [18]. Indeed, PHB degradation leads to D-(-)-3-hydroxy-butyric acid, which is a normal constituent of blood. Although PHB possesses a chemical structure very similar to the synthetic biodegradable polyesters, it degrades very slowly due to its high crystallinity. Therefore, PHB nanoparticles present great potential as depot devices [19]. To reduce its crystallinity and result in materials with better processability, as well as to increase its degradation rate, PHB is often copolymerised with 3-hydroxyvaleric acid (HV). PHB and P(HB-HV) may find applications as tissue engineering scaffolds and drug delivery nanoparticles [20].

\subsubsection{Other synthetic biodegradable polymers}

Polyanhydrides are the most widely investigated biodegradable polymers. Indeed, anhydride bonds (CO-O-CO) are highly sensitive and degrade through hydrolysis into corresponding dicarboxylic acids. The degradation occurs by a surface erosion mechanism, which allows for the release of encapsulated drugs at constant and slow rates. Furthermore, the chemical composition of polyanhydrides can be customised to develop materials with a wide range of degradation kinetics. Aliphatic linear polyanhydrides degrade within a few days, while polyanhydrides containing aromatic groups take longer to degrade, as much as a year [21]. As a consequence, it is possible to control the drug release rate as well as the release pattern. Moreover, polyanhydrides possess a hydrophobic backbone that prevents water penetration which protects the encapsulated drugs from hydrolysis [22].

Aliphatic polycarbonates, such as poly(trimethylene carbonate) (PTMC), are elastomeric polymers with excellent flexibility and softness that make them easy to process. Moreover, sensitive drugs can be encapsulated into polycarbonate-based nanoparticles under mild conditions. PTMC degrades slowly ( $>1$ year) by surface erosion and advantageously does not produce acidic degradation products. Indeed, PTMC degradation leads to 
1,3-propanediol and carbonic acid [13]. PTMC is generally copolymerised with other cyclic lactones and some of the copolymers have been commercialised and are approved by the USFDA for clinical applications [23].

Polyphosphazenes are hybrid organic-inorganic polymers that contain a phosphorus-nitrogen backbone and organic or organometallic side groups that are attached to the phosphorus atoms, which dictate the polymer properties. A huge selection of substituents may be easily introduced by common organic chemistry and the inorganic backbone can degrade by hydrolysis with rates ranging from a few hours to years, depending on the side groups. The degradation leads to neutral products and may have a $\mathrm{pH}$ buffering effect when combined with polyesters [24]. Polyphosphazenes are flexible polymers with good processability and they may participate in noncovalent bonding which in-turn may create new interfacial interactions with biological systems. Consequently, the unique structural diversity of biodegradable polyphosphazenes associated with their potential multifunctionality should allow for the design of nanomaterials with superior biological characteristics [25]. Polyphosphazenes-based nanofibres and nanoparticles show significant promise in drug delivery and tissue engineering applications. Interestingly, organometallic derivatives of polyphosphazenes combine the electronic properties associated with the transition metal anchored to the side groups and the processing advantages of organic polymers. Polyphosphazenes may also be used as coatings on hydrophilic superparamagnetic nanoparticles in order to enhance their water dispersibility and colloidal and chemical stability [26].

Other synthetic biodegradable polymers are also susceptible to hydrolysis. Poly(ortho esters), poly( $p$-dioxanone), poly(propylene fumarate), and poly(ester urethanes) have been used on their own and as copolymers in biomedical applications [27].

\subsubsection{Polysaccharides}

Polysaccharides are found in numerous organisms, such as in mammals, insects, marine organisms and plants. They are the homopolymers or copolymers of monosaccharides, and are very diverse in their chemical structure and composition, molecular weight, and ionic character, leading to various biological activities. Polysaccharides have been widely used in biomedical applications since they are non-toxic, possess a high content of functional groups that can be easily chemically modified, and have excellent properties. Polysaccharide-based biomaterials can be designed as self-assembled micelles, cross-linked nanogels, and fibrous meshes. Accordingly, they are employed for various nanomedicine applications such as in drug delivery carriers, biosensors and tissue engineering scaffolds [28].

Chitosan is a modified polymer prepared by the partial $N$-deacetylation of chitin, which is one of the most abundant natural polymers and found in the 
exoskeletons of insects and arthropods. Its chemical and physical properties depend on its molecular weight, degree of deacetylation, and the distribution of the acetyl groups in the backbone. Chitosan is easier to develop as a biomaterial than chitin due to its better solubility in water and organic solvents. Chitosan is a cationic polymer that possesses polyelectrolyte behaviour and may complex with various negatively-charged biomolecules which enhances its biological activity and makes it a very effective mucoadhesive. Furthermore, it is capable of chelating various metal ions [7]. The structure of chitosan is similar to glycosaminoglycans present in the human body, and it has been shown to elicit a minimal foreign body response and to have a stimulatory influence on immune cells, which may stimulate wound healing processes [3]. Moreover, the cationic structure of chitosan also confers antimicrobial properties, cellular binding capabilities, hemostatic properties, as well as anti-bacterial and anti-fungal properties [29]. Lysozyme and chitosanase degrade chitosan into glucosamine and the in vivo degradation rates range from between weeks to 6 months, depending on the degree of deacetylation [30].

Alginates are unbranched copolymers of $\beta$-D-mannuronic acid (M) and $\alpha$-L-guluronic acid (G), existing as mixed salts of calcium, magnesium, sodium and potassium. They are found in brown algae and their physicochemical properties depend on the proportion and the distribution of $\mathrm{M}$ and $\mathrm{G}$ monomers which are intrinsically linked to the source origin [31]. Alginates do not enzymatically degrade in vivo since alginate lyases are found in algae and marine microorganisms, and they do not possess bioactive sequences which may be recognised by cells [30]. However, alginates are abundant and are relatively low in cost. They show excellent biocompatibility, low toxicity, and non-immunogenicity. They also possess high functionality and they may easily form hydrogels through simple gelation with divalent cations [29]. Moreover, alginates can electrostatically interact with oppositely charged chitosan and poly(L-lysine) to develop hydrophilic nanocarriers with high potential as vectors in biomedical and pharmaceutical applications [32,33].

Dextran is produced from sucrose through bacteria or yeast fermentation. It consists essentially of $\alpha-1,6$ linked glucopyranoside residues with a small percentage of $\alpha-1,3$ linked residues. The length and arrangement of branches differ depending on the enzyme's bacterial source. Dextran is cleaved by microbial dextranases and slowly degrades in comparison with other polysaccharides. Due to its high content of hydroxylic groups, dextran may be easily functionalised [34]. Additionally, it is a natural analogue of poly(ethylene glycol) (PEG) and therefore dextran derivatives are used in a wide range of biomedical applications because of their excellent solubility in aqueous solutions, biocompatibility and nonfouling properties. Dextran-based hydrogels can also be formed by physical or chemical crosslinking. Recently, dextran-based nanogels have been developed to act as cell tracking probes for advanced in vivo imaging techniques [35]. 


\subsubsection{Glycosaminoglycans}

Glycosaminoglycans are linear, anionic, naturally occurring polysaccharides primarily composed of glucuronic acid, iduronic acid and $\mathrm{N}$-acetylgalactosamine with variable sulfation patterns, which is critical for biological activities. Indeed, the sulfation content plays a major role in glycosaminoglycan-protein binding. As the synthetic development of glycopolymers with high reproducibility and yield has yet to be achieved, the use of natural glycosaminoglycans is of interest for the design of bioactive biomaterials. Heparin is one of the most studied glycosaminoglycans due to its high negative charge density which can be attributed to the presence of approximately 2.7 sulfate groups per disaccharide unit. Such high negative charge density enables interactions with several proteins. Heparin has mainly been used as a surface coating to functionalise a variety of nanomaterials [36].

Hyaluronic acid (HA) is the most commonly used carbohydrate-based natural polymer in tissue engineering. It is composed of D-glucuronic acid and $\mathrm{N}$-acetyl-D-glucosamine. HA is the only non-sulfated glycosaminoglycan and in contrast to other glycosaminoglycans, it does not covalently bond to proteins. $\mathrm{HA}$ is synthesised at the inner wall of the plasma membrane and is present in the extracellular matrix, connective tissues and synovial fluids. It is the most abundant natural polymer present in the human body and more than $50 \%$ is found in the skin, lung and intestine. Commercial HA is produced from animal tissues and microbial fermentations [37]. HA can be obtained in a wide range of molecular weights, up to $10^{7} \mathrm{~g} \mathrm{~mol}^{-1}$, which allows HA to assume a variety of roles within the body. Indeed, high-molecular-weight HA is considered anti-angiogenic and non-immunogenic and plays a role in maintaining cell integrity, while low-molecular-weight HA is considered inflammatory, immuno-stimulatory and angiogenic and induces receptor-mediated intracellular signalling. In vivo, $\mathrm{HA}$ is enzymatically degraded by hyaluronidase, $\beta$-D-glucuronidase, and $\beta$ - $N$-acetyl-hexosaminidase at a high degradation rate (from hours to 1 month). HA degradation leads to glucuronic acid and $\mathrm{N}$-acetylglucosamine, which are finally metabolised to carbon dioxide, water and urea [30,38]. Because of its excellent biocompatibility and physicochemical properties, HA has been widely used in wound dressings, drug delivery applications and for tissue engineering including cartilage, liver, vascular, dermal, ophthalmic and nerve repair or regeneration. However, the commercially available HA homopolymer is too mechanically weak to be used as a supportive scaffold and its half-life is too short for long-term clinical applications. As a consequence, HA is often chemically modified or cross-linked to allow for the development of nanofibres, nanogels or self-assembled nanoparticles $[29,37,39]$. Moreover, it was demonstrated that when sulfur-modified, HA shows a higher binding affinity to growth factors than heparin [36]. 


\subsubsection{Proteins}

Proteins are essentially high-molecular-weight amino acid polymers arranged in a three-dimensional folded structure that are naturally degraded by a wide range of proteases. Proteins display a wide range of biological functions in nature which has motivated a significant amount of interest in the development of protein-based biomaterials. Collagen accounts for about a fourth of the total protein content of the human body. It is a fibrous protein that maintains the structural integrity of the extracellular matrix in tissues. Its primary structure is a polypeptide chain composed of repeating triplets of glycine-X-Y, where $\mathrm{X}$ and $\mathrm{Y}$ are typically proline and hydroxyproline. There are 28 types of collagen molecules and collagen type I (present in the skin, tendons and bones), represents $90 \%$ of all collagen types [40]. Collagen-based nanofibres have been applied for wound dressings and preliminary vascular tissue engineering. However, commercial sources of collagen type I are generally derived from rat tail, bovine dermis, or human placenta. Therefore, recombinant systems have been developed due to the risks associated with infectious disease transmission from allogenic or xenogenic materials, the potential for immunogenicity, as well as the high cost of purification, quality concerns, and product homogeneity for mass production [30].

Gelatin is obtained by the denaturation and physicochemical degradation of collagen and its properties depend on the production process. Gelatin consists of 19 amino acids and is arranged in single-stranded molecules. It possesses similar hemostatic properties to its collagen precursor and is enzymatically degraded by collagenases [41]. Gelatin is soluble in aqueous solutions; however it possesses cationic and anionic groups coupled with strong hydrogen binding ability, which can make fibre-forming a challenge. Many molecules have been successfully encapsulated into gelatin-based nanoparticles, and the mechanical stability of nanofibre-based scaffolds may be enhanced by chemical crosslinking with various agents $[6,40]$.

Silk is a natural protein mainly produced by silkworms and spiders. Silk fibre possesses a crystallised and compact structure consisting of hydrophobic fibroin as the core protein coated by hydrophilic sericin, which maintains the physical structure of fibroin. The presence of sericin is the main concern in terms of usefulness for biomedical applications, since it is associated with hypersensitivity reactions and poor biocompatibility. When sericin is removed, or absent, like in silk fibres from spiders, the immune response is similar to that of other biomaterials [30]. Fibroin is highly biocompatible, biodegradable, induces a limited inflammatory response, and possesses excellent mechanical properties. Moreover, silk-based biomaterials have high thermal stability over a wide range of temperatures (up to $250^{\circ} \mathrm{C}$ ) and therefore they can be sterilised by autoclave. Silk degradation occurs through enzymatic proteolysis from enzymes such as chymotrypsin, actinase, and carboxylase. The degradation rate in vivo can be tailored from months to years based on many 
factors, such as the silk processing conditions and the physical characteristics of the material. Silk-based biomaterials have been widely processed as nanofibre-based scaffolds for tissue engineering applications and wound dressings $[40,42]$.

\subsection{POLYMERIC NANOFIBRES AND NANOFIBROUS SCAFFOLDS}

Polymeric nanofibres have been developed for a variety of applications. In the medical field, the market of nanofibre-based materials is very nascent and therefore research in this area is rapidly expanding [43]. The potential medical applications are in the development of scaffolds for tissue engineering, carriers of bioactive compounds and cells, and in wound dressings. Additionally, in-terms of positively promoting cell-polymeric matrix interactions, the high surface area of the nanostructured, nanofibrous scaffolds allows for oxygen permeability and provides sufficient space for nutrient and waste exchange [44]. Moreover, in wound dressings, fluid accumulation at the wound site is limited and the material pore size prevents bacterial penetration. When used as carriers, nanofibrous materials may offer site-specific delivery of multiple drugs, genes and growth factors. Furthermore, their morphology and porosity can be modulated to control the molecule release profile [45]. Finally, the nanofibre-based scaffold surface can easily be functionalised by chemical or physical methods in order to carry various functionalities.

\subsubsection{Fabrication}

As illustrated in Figure 2, three main techniques are used to fabricate nanofibres based on biodegradable polymers: electrospinning, thermally-induced phase separation and self-assembly. 


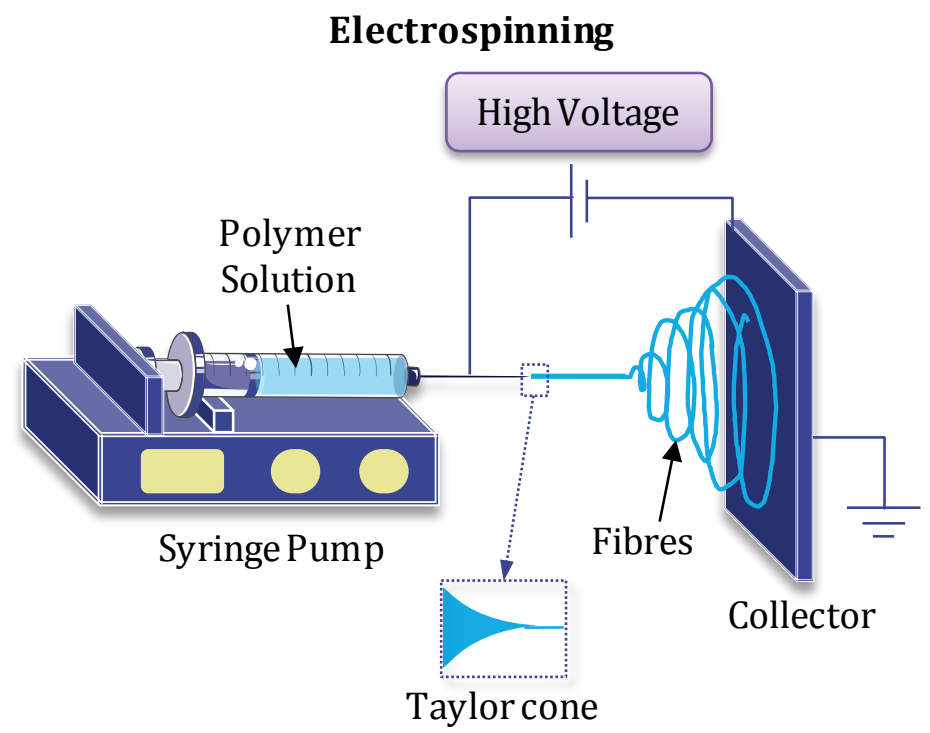

Thermally-Induced Phase Separation (TIPS)

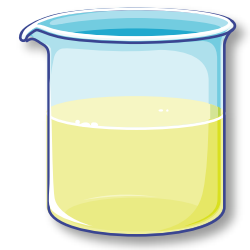

Polymer

Solution

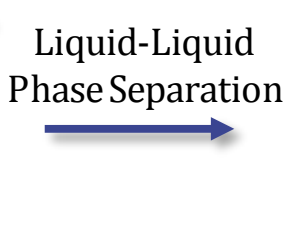

\section{Liquid-Liquid}

Phase Separation

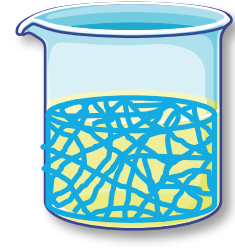

Polymer

Gelation

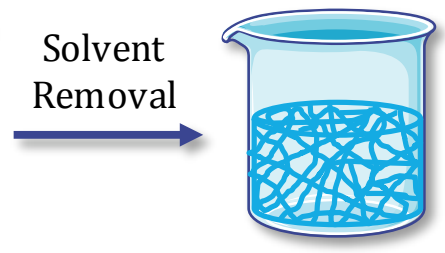

Nanofibrous

Scaffold

\section{Self-assembly}

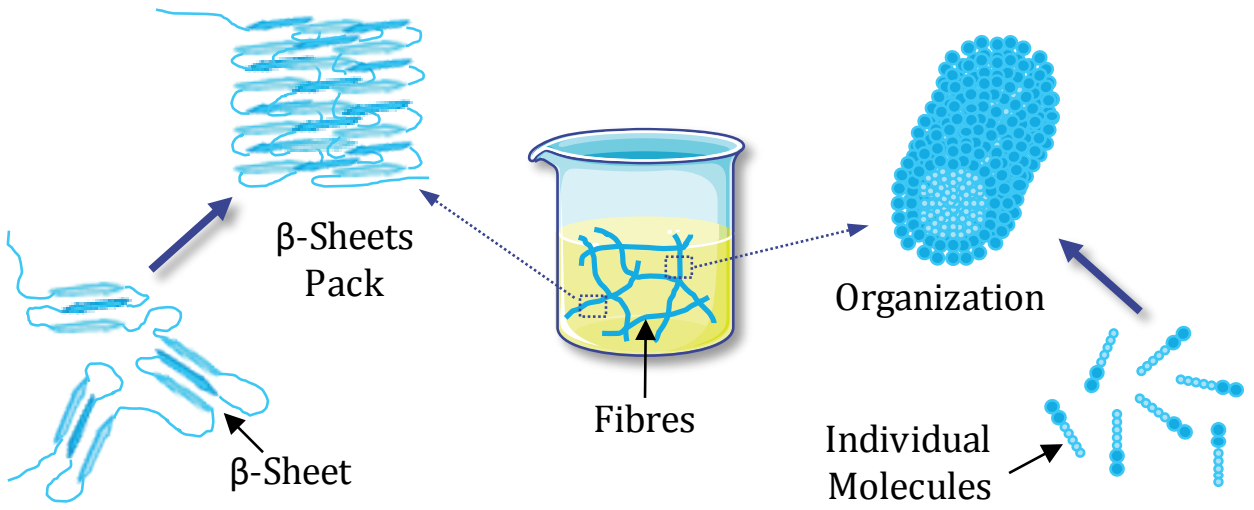

Figure 2. Main techniques used for the development of polymeric nanofibres 
Among the three, electrospinning is the most widely studied technique. It is a simple, cost-effective, robust, and versatile process capable of producing polymeric fibres from a variety of polymer melts and solutions, with nanoscale diameters ranging from 50-1000 nm or greater. The electrospinning setup includes a high voltage power supply between a polymer solution or melt reservoir and a grounded collector (Figure 2). Due to the electric field, the polymer solution surface at the end of the spinneret is electrically charged, with even charge distribution over the surface. Under the influence of electrostatic interactions, an increase in the electrical potential leads to a drop distortion into a so-called "Taylor cone". When the applied electric field reaches a critical value, the repulsive electrical forces overcome the surface tension forces and a jet is ejected continuously from the tip of the Taylor cone. Eventually, the electrified jet is attracted by the grounded collectors of opposite polarity placed under the spinneret. The space between the spinneret tip and the collector allows for solvent evaporation and therefore a solid polymeric non-woven fibrous matrix is deposited on the surface of the collector [46]. Conventional electrospinning produces randomly oriented nanofibres; however, aligned electrospun fibres (uniaxially aligned, radially aligned or in a wavy form) can also be obtained when using rotating and dual collectors or by manipulating the electrical field. Finally, the flat matrix may be stacked, folded, wound and twisted to fabricate three dimensional scaffolds with various shapes such as sheets, tubes or threads [47]. The morphology of the fibrous matrix is influenced and controlled by the polymeric solution parameters (viscosity, conductivity, surface tension), processing parameters (electric field, distance between the spinneret and the collector, flow rate and spinneret diameter) as well as ambient parameters (temperature, humidity). Indeed, the polymer nature determines the rate of degradation, while the solution and processing parameters determine the nanofibre diameter and the amount of polymeric beads that may be formed along the fibre [5].

Thermally-induced phase separation (TIPS) is a relatively simple procedure with very minimal requirements in terms of equipment. The TIPS approach allows for great processing flexibility with overall shape and pore structure control, as well as allowing for the design of extracellular matrix-like nanofibres ranging from $50-500 \mathrm{~nm}$. Moreover, TIPS can be combined with other techniques to create simultaneous nano- and macro- architectures through the formation of macroporosity in the nanofibrous matrix, and to fabricate nanofibrous hollow microspheres without the need for a template [48-50]. The TIPS method involves five basic steps: polymer dissolution, liquid-liquid phase separation process, polymer gelation, solvent extraction, and eventually freezing and freeze-drying under vacuum (Figure 2). TIPS is based on the thermodynamic instability of a homogenous solution of polymer in solvent which, by cooling the solution below the polymer glass transition temperature, will spontaneously separate into two phases: polymer-rich and polymer-lean phases. Upon extraction and freeze drying, the polymer-rich 
phase solidifies to form the polymer skeleton and eventually leaves behind a solid polymeric scaffold whose morphology is affected by processing variables such as the polymer nature, concentration, solvent, and temperature. Therefore under the right conditions, nanofibrous scaffolds may be developed. Actually, gelation is the step that controls the porous morphology since low gelation temperature leads to nanoscale-fibre scaffolds, whereas high gelation temperature leads to a platelet-like structure due to crystal nucleation and growth. However, the fibre average diameter is not significantly affected by gelation conditions or polymer concentration [5].

Self-assembly involves the spontaneous organisation of individual molecules into an ordered structure or pattern through non-covalent interactions such as hydrogen bonding, van der Waals forces, electrostatic forces, or hydrophobic forces (Figure 2). The goal of self-assembly is the formation of thermally stable protein-like molecular architectures, through the creation of nanofibres from synthesised small molecules and oligopeptides with a well-defined chemistry. For instance, oligopeptides consist of alternating hydrophilic and hydrophobic amino acids forming stable $\beta$-sheet structures. When an aqueous peptide solution is added to a physiological salt-containing solution, $\beta$-sheets pack together to form double-layered $\beta$-sheet nanofibres, without the need for temperature changes [51]. Self-assembly is a simple process for nanofibre fabrication. It can also be used to easily encapsulate cells in a hydrogel and can be used in an injectable form for in situ scaffold formation. Control of self-assembly in the design of nanofibres is carried out by switching the $\mathrm{pH}$, through the introduction of divalent ions, and by varying the temperature and the concentration. However, self-assembly is limited by the choice of molecules whose synthesis is time-consuming and quite expensive. Self-assembly also leads to nanofibres in a gel-form, while electrospinning and TIPS give nanofibres in a dehydrated-form. Moreover, the self-assembly process does not easily allow for the control of pore size and shape within the hydrogel, and leads to small fibre diameters in comparison to those fabricated by other techniques. Indeed, individual fibre diameters are around 10-20 nm, and fibre lengths can only reach several micrometers [49].

\subsubsection{Properties}

The process and the processing parameters have a significant effect on the geometric properties of the nanofibres obtained, which determine the physical and mechanical properties of the polymeric materials. Some examples of polymers and solvents used for designing nanofibres by electrospinning and TIPS, associated to the fibre diameters, are given in Table 1. 
Table 1. Examples of nanofibre diameters in correlation with solvent, polymer nature and concentration, obtained by electrospinning and TIPS

\begin{tabular}{|c|c|c|c|c|c|}
\hline Polymer & Solvent ${ }^{\mathrm{a}}$ & $\begin{array}{l}\text { Conc. }^{b} \\
\text { (w/v\%) }\end{array}$ & $\begin{array}{c}F^{c} \\
(n m)\end{array}$ & $\begin{array}{c}\text { FL/FD } \\
\text { ratio }^{\mathrm{d}}\end{array}$ & Ref. \\
\hline \multicolumn{6}{|c|}{ Electrospinning } \\
\hline \multirow{3}{*}{ PCL } & $\mathrm{CHCl}_{3}$ & 10 & 4173 & & \\
\hline & $\mathrm{FA} / \mathrm{CHCl}_{3}$ & 10 & 222 & - & {$[55]$} \\
\hline & FA/AA & 10 & 266 & & \\
\hline PDLLA & TFE & 20.8 & 750 & - & [70] \\
\hline PCL/PLLA & $\mathrm{CHCl}_{3} / \mathrm{MeOH}$ & $8-11$ & $347-1340$ & - & [54] \\
\hline PCL/PTMC & DCM/DMF & 8 & $203-302$ & - & [66] \\
\hline PHB & $\mathrm{CHCl}_{3} / \mathrm{DMF}$ & 14 & 950 & - & [18] \\
\hline $\mathrm{P}(\mathrm{HB}-\mathrm{HV})$ & $\mathrm{CHCl}_{3}$ & 18 & 571 & - & [71] \\
\hline Polyphosphazene & $\mathrm{CHCl}_{3}$ & 7 & 1200 & - & {$[72]$} \\
\hline Chitin & HFIP & 6 & 110 & - & [73] \\
\hline Chitosan & $\mathrm{H}_{2} \mathrm{O} / \mathrm{AA}$ & 7 & $40-130$ & - & [73] \\
\hline Alginate/PEO & $\mathrm{H}_{2} \mathrm{O}$ & 10.6 & $80-300$ & - & [74] \\
\hline Dextran & $\mathrm{H}_{2} \mathrm{O}$ & 75 & 186 & - & [75] \\
\hline $\mathrm{HA}$ & $\mathrm{HCl}$ & 1.3 & $49-74$ & - & [76] \\
\hline Collagen & HFIP & 8 & $100-1200$ & - & [63] \\
\hline Gelatin & $\mathrm{H}_{2} \mathrm{O} / \mathrm{AA}$ & 10 & 110 & - & [77] \\
\hline \multirow[t]{2}{*}{ Silk } & HFA & $3-7$ & $250-500$ & - & [42] \\
\hline & & TIPS & & & \\
\hline PLLA & THF & $1-7.5$ & $164-169$ & $6.1-12.5$ & [60] \\
\hline PCL/PLLA & THF & 5 & $95-175$ & - & [78] \\
\hline PLGA & THF & $5-12$ & $259-569$ & $3.1-11.7$ & [79] \\
\hline PHB & $\mathrm{CHCl}_{3} / \mathrm{DIOX}$ & $1-5$ & $161-170$ & $4.8-8.0$ & [61] \\
\hline Gelatin & $\mathrm{MeOH} / \mathrm{H}_{2} \mathrm{O}$ & $5-10$ & 157-177 & $3.2-6.7$ & [50] \\
\hline
\end{tabular}

a Solvent abbreviations: AA: acetic acid, $\mathrm{CHCl}_{3}$ : chloroform, DCM: dichloromethane, DMF: $N, N$-dimethylformamide, DIOX: dioxane, FA: formic acid, $\mathrm{H}_{2} \mathrm{O}$ : water, $\mathrm{HCl}$ : hydrochloric acid, HFA: hexafluoroacetone, HFIP: 1,1,1,3,3,3-hexafluoro-2-propanol, MeOH: methanol, TFE: 2,2,2-trifluoroethanol, THF: tetrahydrofuran

${ }^{b}$ Conc.: polymer concentration

c FD: fibre diameter

d FL/FD ratio: fibre length/fibre diameter ratio 
Electrospun nanofibre diameters may be increased by increasing the flow rate, the spinneret tip diameter and its distance from the collector, and decreasing the voltage and the solution charge density. Moreover, the viscosity of the solution impacts on the uniformity of the bead-free nanofibres [52]. Indeed, there is an optimal feed solution viscosity for electrospinning since no continuous fibres may form at very low viscosity, whereas the ejection of jets from highly viscous polymer solutions is very difficult. In the optimal range, a more viscous feed solution, due to the higher polymer concentration and molecular weight, leads to larger and more uniform fibre diameters with a reduction in the number of beads along the fibres [53]. For instance, PCL/PLLA nanofibres electrospun from a chloroform/methanol solution had dense bead structures with a smaller diameter for low concentrations of PCL/PLLA, in comparison with a concentration of $11 \mathrm{w} / \mathrm{v} \%$ which resulted in fibres almost four times larger with bead-free structures (Table 1) [54]. Van der Schueren et al. have demonstrated the importance of the solvent during the electrospinning process. Indeed, different solvents lead to different polymer solution surface tensions, and a high surface tension tends to inhibit the electrospinning process. For instance, using chloroform as the solvent led to uniform PCL fibres in the microscale range, while the binary system of formic acid/chloroform resulted in smaller fibres in the nanoscale range with noticeable beads. When using the formic acid / acetic acid system, the number of beads drastically decreased (Table 1). The composition of the binary system also impacted on the eventual fibre diameter. By increasing the amount of acetic acid from $10 \mathrm{v} \%$ to $80 \mathrm{v} \%$, the average fibre diameter showed a minor increase (from 545 to $662 \mathrm{~nm}$ ), though this was associated with a large increase in the standard deviation (from 80 to $420 \mathrm{~nm}$ ). This trend was attributed to changes in the solution conductivity [55]. Porous nanofibres can also be fabricated by inducing phase separation between the polymer and the solvent, which can be induced through temperature reduction or by using a highly volatile solvent $[56,57]$. Finally, the composition of fibres can be easily tailored by using different polymers, composite materials and encapsulations during electrospinning, which may also lead to changes in the nanofibre diameters. Khatri et al. have demonstrated that the fibre diameter decreased from 860 to $715 \mathrm{~nm}$ with increasing PLLA ratio in PCL/PLLA blends [58]. When magnetite nanoparticles were encapsulated into the PCL nanofibres during the electrospinning process, the nanofibre diameter decreased from $864 \mathrm{~nm}$ to $202 \mathrm{~nm}$ through the inclusion of up to $15 \%$ of magnetite nanoparticles, which can be attributed to changes in the solution electrical conductivity and viscosity. Nevertheless, the addition of $20 \%$ of magnetite nanoparticles increased abruptly the fibre diameter to $664 \mathrm{~nm}$. This phenomenon was explained by the agglomeration of magnetite nanoparticles resulting in a too high solution viscosity and a low electrospinnability [59].

The nanofibrous structure is also affected by the processing parameters when using the TIPS method for nanofibrous scaffold preparation (Table 1). The 
solvent and the gelation temperature have a huge impact on the morphology of the foam. Indeed, if the polymer / solvent mixture is cooled fast to a low temperature that allows the solvent to freeze into a solid state, a solid-liquid phase separation will take place instead of the liquid-liquid phase separation. As a consequence, channel and ladder-like features are observed instead of nanofibrous networks. However, when the polymer/solvent mixture is appropriate, nanofibrous structures are obtained even at low gelation temperatures. Ma et al. have demonstrated the peculiarity of the PLLA/THF system, where nanofibrous scaffolds were obtained only with temperatures of below $15{ }^{\circ} \mathrm{C}[60]$. Li et al. reported that THF and DMF could not produce PHB nanofibrous structures, while nanofibrous scaffolds were obtained by using a chloroform / dioxane mixture and gelation temperature of below $4{ }^{\circ} \mathrm{C}$ [61]. Moreover, the authors have found that the average fibre diameter was not affected by the gelation temperature; however the interfibre spacing, which can be related to the fibre length / fibre diameter ratio, decreased and became more uniform at lower gelation temperatures. The same trend is observed when the polymer concentration was increased $[60,61]$. In this way, the porosity of the scaffold may be controlled.

Mechanical characterisation of nanofibres and nanofibrous scaffolds is crucial for tissue engineering applications because the scaffold must provide a mechanically stable support for cell development, and must be able to withstand the forces exerted by growing tissues and physiological activities. Some mechanical properties of polymeric nanofibres and nanofibrous scaffolds obtained by electrospinning and TIPS are given in Table 2 . The values are different from those obtained in equivalent bulk materials [27]. Indeed for fibre-based materials, the mechanical properties are affected by the polymer molecular weight, morphology, crystallinity, as well as the material size and shape such as porosity, pore area and fibre size, density and orientation [44]. For instance, when electrospun fibre-based materials are developed with aligned fibres, the modulus and the tensile strength increase and the mechanical behaviour becomes anisotropic in comparison to randomly arranged fibres $[62,63]$. The fibre size also affects the mechanical properties as a reduction in the size improves the orientation and decreases the quantity of defects in the structure. As a consequence, a higher modulus and strength are generally obtained. The mechanical behaviour of electrospun fibre-based materials may also be tailored by: applying thermal post-treatments, using polymer blends, crosslinking the fibres, modifying the fibre surface, as well as encapsulating nanoparticles [44]. Ramier et al. have demonstrated that the incorporation of hydroxyapatite nanoparticles within PHB nanofibres increased the elastic modulus and the tensile strength by $67 \%$ and $51 \%$, respectively. In contrast, when the hydroxyapatite nanoparticles were sprayed over the PHB nanofibres during the electrospinning process, the mechanical properties drastically decreased. This can be attributed to the higher porosity of the scaffold due to weaker interactions between the fibres and the 
hydroxyapatite nanoparticles [18]. Nanofibrous scaffolds obtained by TIPS exhibit significantly better mechanical behaviour than solid-walled porous scaffolds [50]. Moreover, the mechanical properties can be tailored by using polymer blends and by varying the polymer concentration in the gelling solution $[60,61]$. Indeed, an increase in the polymer concentration leads to a higher network density associated with lower porosity. As a consequence, the Young's modulus and the tensile strength both increase [60].

Table 2. Examples of mechanical properties of some polymeric nanofibres and nanofibrous scaffolds obtained by electrospinning and TIPS

\begin{tabular}{ccccc}
\hline Polymer & $\begin{array}{c}\text { Young's } \\
\text { Modulus } \\
(\mathrm{MPa})\end{array}$ & $\begin{array}{c}\text { Tensile } \\
\text { Strength } \\
(\mathrm{MPa})\end{array}$ & $\begin{array}{c}\text { Strain at } \\
\text { Break } \\
(\%)\end{array}$ & Ref. \\
\hline Electrospinning & & \\
\hline PCL & $6-60$ & $0.6-40$ & $0.6-7.7$ & {$[46,59,65,80]$} \\
\hline PDLLA & $13.9-70$ & 1.1 & 1.8 & {$[65,70]$} \\
\hline PLLA & $10-66$ & $0.1-7.8$ & $1.8-127$ & {$[40,65]$} \\
\hline PLGA & $30-140$ & $1.8-45$ & 2 & {$[62,65]$} \\
\hline PHB & 238 & 10.7 & 7.3 & {$[18]$} \\
\hline Chitosan & 155 & 4.07 & 0.12 & {$[81$} \\
\hline Collagen & $80-262$ & $3-12$ & - & {$[40,46,63]$} \\
\hline Gelatin & $105-499$ & $2.3-12$ & $10.6-96$ & {$[40,46]$} \\
\hline PLLA & $4-20$ & TIPS & & {$[60]$} \\
\hline PHB & 40 & $0.15-0.6$ & $6-9$ & {$[61]$} \\
\hline
\end{tabular}

The physical properties of the nanofibrous scaffolds are crucial as they influence the degradation behaviour of the material as well as the biological properties. Indeed, it was found that the fibre diameter influences the cell spreading proliferation, migration and differentiation $[40,64]$. Cells migrate poorly into small-diameter fibre scaffolds and readily penetrate into large-diameter fibre scaffolds. However, cells are restricted to spreading along single fibres for large-diameter fibres, whereas cells are guided by the underlying fibrous matrix for small-diameter fibres. As a consequence, cell differentiation is more extensive on fibres with smaller diameters. Moreover, cells are also affected by the fibre alignment. Since cells adhere and elongate along the fibre axis, a more organised deposition of extracellular matrix is obtained in aligned fibre-based scaffolds. As a consequence, the resulting tissues possess higher stiffness and modulus in comparison with tissues formed by cells seeded onto random fibre-based scaffolds [64]. Finally, cells have a higher proliferation rate on scaffolds that are more stable because 
scaffolds lose their porosity and structural integrity when degrading, thus preventing cell adhesion and ingrowth [65]. The degradation behaviour of macroscale degradable polymers has been comprehensively studied and was found to depend on a wide range of parameters, such as the polymer composition, molecular weight, crystallinity, porosity, material size and shape. However, it is important to note that the degradation rate of polymers is different between the bulk material and the nanofibre-based scaffolds. For some polymers, the degradation rate of larger structures is faster than that of nanofibres due to autocatalysis in larger structures [66]. Nevertheless, the hydrolytic degradation was found to be much more rapid for nanofibrous scaffolds obtained by TIPS in comparison to solid-walled scaffolds, even if the wettability in the nanofibrous scaffold is smaller because of small interfibre spacing and the large amount of relatively-hydrophobic surface area. Indeed, the high amount of surface area offers more available sites for polymer hydrolysis. Besides, fibre aggregation occurs during the degradation leading to a decrease in the surface area of the nanofibrous scaffolds, which consequently induces a reduction in the degradation rate with time [67]. Fibre aggregation during degradation is also observed for electrospun nanofibres that are prepared from polymers with glass transition temperatures lower than the degradation temperature. For some polymers, the degradation results in fibre swelling. The fibres may also change from smooth and straight to coil and wavy [65]. For rigid and crystalline polymers associated with slow degradation rates, the fibres tend to break along the fibre axis and the broken ends are more susceptible to hydrolytic attacks [68]. Some attempts to reduce the degradation rate have led to the design of nanofibres with various polymer blends as well as composite nanofibres with encapsulated apatitic nanoparticles. Indeed, Ji et al. demonstrated that the incorporation of apatite nanoparticles within PLGA/PLC electrospun fibres had a buffering effect as the nanoparticles neutralised the acidic degradation products that are generated upon polymer degradation, hence slowing down the scaffold degradation [69].

\subsubsection{Applications of nanofibrous materials}

The ultimate goal of tissue engineering approaches is to successfully repair and restore the function of damaged or diseased tissues. An important feature of tissue engineering is the design of polymeric scaffolds that may be used as carriers for therapeutic cell delivery to the defect region, or may act as space fillers that would recruit surrounding cells and allow tissue development. As a consequence, compelling requirements have been identified for scaffold design: (1) biocompatibility and biodegradability with an appropriate degradation rate; (2) adequate morphology; (3) multi-scale interconnected porous structure; (4) optimal mechanical strength; (5) surface properties that could regulate appropriate cell activities [82]. Various scaffolds have been prepared using many different methods including particulate leaching, textile technologies, and phase separation [83]. Although these scaffolds exhibit 
certain advantages, their pore sizes and fibre diameters are often micron-scale, far from the natural nano-scale of extracellular matrix. As a consequence, cells attach and spread in a flatten pattern and still exhibit a two dimensional topography, similar to if they had been cultured on 2D flat surfaces [84]. In addition to the architectural similarity with natural extracellular matrix, nanofibrous and nanofibre-based scaffolds can absorb more proteins due to their high surface area and porosity, and therefore present more binding sites to cell membrane receptors. As a consequence, they may enhance cell adhesion and provide an excellent micro/nano environment for cell proliferation and activity [85]. Since a number of natural and synthetic biodegradable polymers have been successfully fabricated as nanofibres, nanofibrous scaffolds have been explored for many tissue engineering applications. Thus, they have been investigated for use in bone regeneration as well as soft tissue regeneration, such as for the regeneration of cardiovascular tissue, cardiac grafts, nerve, cartilage, ligament and skin $[5,42,86]$. Therefore, numerous studies have investigated the behaviour of various cells, including cardiomyocytes, chondrocytes, keratinocytes and stem cells seeded over nanofibrous materials $[61,65,85,87]$. In wound healing applications, nanofibre-based materials possess many advantages. Indeed, they help generate dressings with good oxygen permeation, sufficient drainage of wound exudates, and facilitate the protection of the wound from infection and dehydration. Moreover, they may accelerate the healing process by promoting the migration of cells on the wound surface, and may also reduce wound contraction leading to a decrease in patient morbidity [77,85]. Finally, due to the ease of nanoparticle incorporation within the nanofibres, antimicrobial wound dressings may be further developed [70].

Polymeric nanofibres may also be used to encapsulate and deliver bioactive hydrophilic and hydrophobic molecules for therapeutic applications. The solubility and compatibility of the drug in a drug/polymer/solvent system are decisive factors for the elaboration of nanofibrous carriers, since the system is aimed to deliver a sufficient amount of a drug for an adequate period of time and has to avoid the degradation of drugs during the fabrication process [88]. A wide range of therapeutic molecules, including drugs, proteins, genes and growth factors, have been successfully encapsulated within fibre structures, physically coated or chemically attached on their surfaces $[89,90]$. For instance in the electrospinning process, drug loading can be achieved through various techniques such as; post-spinning modifications that prevent the drug from exposure to the electrospinning process; direct electrospinning of drug/polymer blends that boasts the advantage of being a single-step method; and co-axial or emulsion electrospinning which can be used to develop core-shell morphologies that contribute to prolonged release [47]. Compared with other formulations, electrospinning also offers a high loading capacity. As a matter of fact, the high surface area of nanofibres allows for fast and efficient solvent evaporation limiting drug crystallisation, and therefore leading to the 
formation of amorphous dispersions. Moreover, due to the flexibility of the nanofibre processing, a variety of fibre compositions, porosities and structural architectures may be developed in order to control the drug eluting profiles and to meet the needs of the targeted physiological environment. Indeed, the release mechanism is highly dependent on the distribution of the drug molecules in the fibres as well as the fibre morphology. In this way, the drug release pattern can be tailored by varying the drug travelling distance and diffusion pathway, which are directly related to the polymer degradation mechanism and the drug/polymeric matrix affinity [90]. The principal advantage of nanofibrous carriers over cast-films is the increased drug release, prolonged availability and site-specific delivery into the body, thus achieving high local bioactivity and low systemic side effects. Furthermore, simultaneous administration of multiple drugs may be achieved by encapsulating various therapeutic agents within the same nanofibrous carrier. To ensure the independent controlled release of each drug, sequential electrospinning has been developed to obtain multilayered membranes consisting of various drugs and basement nanofibres [88]. It has also been demonstrated that the encapsulation of drugs into nanospheres which have been subsequently incorporated within nanofibrous scaffolds, reduces the burst release and allows for drug delivery over a prolonged duration [45]. Biodegradable polymeric nanofibre-based carriers can be applied for the prevention of postsurgical adhesions and infections, local chemotherapy, transdermal drug delivery, and tissue engineering $[45,47]$.

\subsection{POLYMERIC NANOPARTICLES}

Polymeric nanoparticles (PNPs) are defined as particulate dispersions or solid particles of 10-1000 nm in size. Within these PNPs, therapeutic agents (such as drugs, DNA, proteins, etc.) as well as fluorescent labels can be dissolved, entrapped, encapsulated or can be attached to the nanoparticle matrix. Two conformations of polymeric nanoparticles are known to exist (Figure 3). The term "nanocapsule" is used when the polymer forms a core, in which a molecule of interest can be entrapped. The other common structure is called nanosphere and refers to a nanoparticle made of entangled polymer chains in which the molecule of interest is present. It is important to note that the molecule can also be absorbed or covalently attached onto the surface of the nanoparticles. 

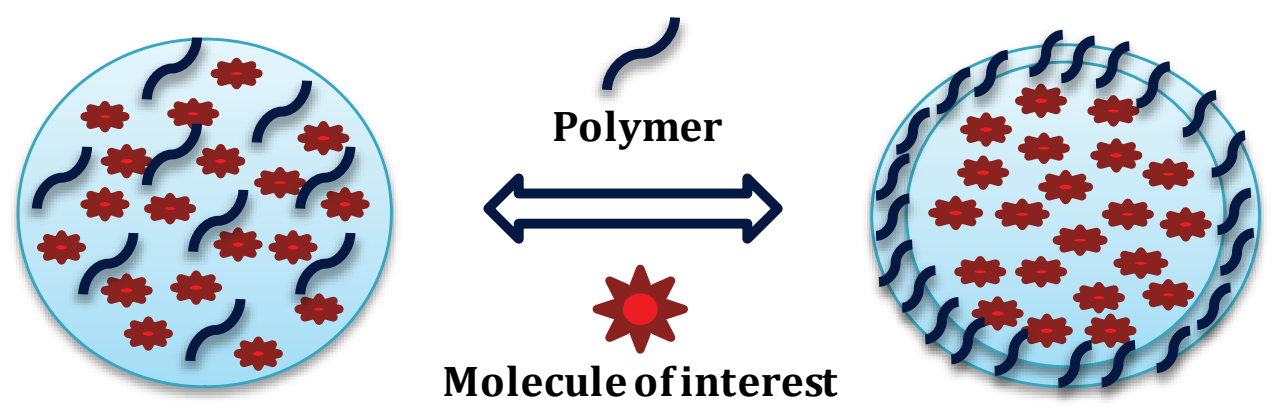

Figure 3. Schematic representation of a nanosphere (left) and a nanocapsule (right)

\subsubsection{Advantages and applications of polymeric nanoparticles}

There has been considerable research interest in using particulate delivery systems as carriers for small and large molecules in drug delivery. Particulate systems, such as nanoparticles, have been used as a physical approach to alter and improve the pharmacokinetic and pharmacodynamic properties of various types of drug molecules. Indeed, the nanometre-size promotes effective permeation through cell membranes and stability in the blood stream. Polymeric nanoparticles have been extensively studied as particulate carriers in the pharmaceutical and medical fields, because they show promise as drug delivery systems. This potential can be attributed to a number of factors including: their controlled and sustained release properties [91,92], subcellular size, which allows for relatively higher intracellular uptake compared to other particulate systems [93], possible improvement of the active substance stability [94], and biocompatibility with tissues and cells when synthesised from materials that are either biocompatible or biodegradable [95]. Polymers are very convenient materials for the manufacture of countless and varied molecular designs that can be integrated into unique nanoparticle constructs with many potential medical applications [12]. Other advantages of nanoencapsulated systems as active substance carriers include: high drug encapsulation efficiency due to optimised drug solubility in the core, low polymer content compared to other nanoparticulated systems such as nanospheres, drug polymeric shell protection against degradation factors such as $\mathrm{pH}$ and light, and the reduction of tissue irritation due to the polymeric shell (Scheme 1) $[96,97]$. As a consequence, PNPs have been extensively studied as drug carriers in the pharmaceutical field $[98,99]$ and different research teams have published reviews about the nanoparticle formation mechanisms [91-101], the classification of nanoparticulated systems [98], and the techniques employed for the preparation of nanocapsules $[96,102]$. 


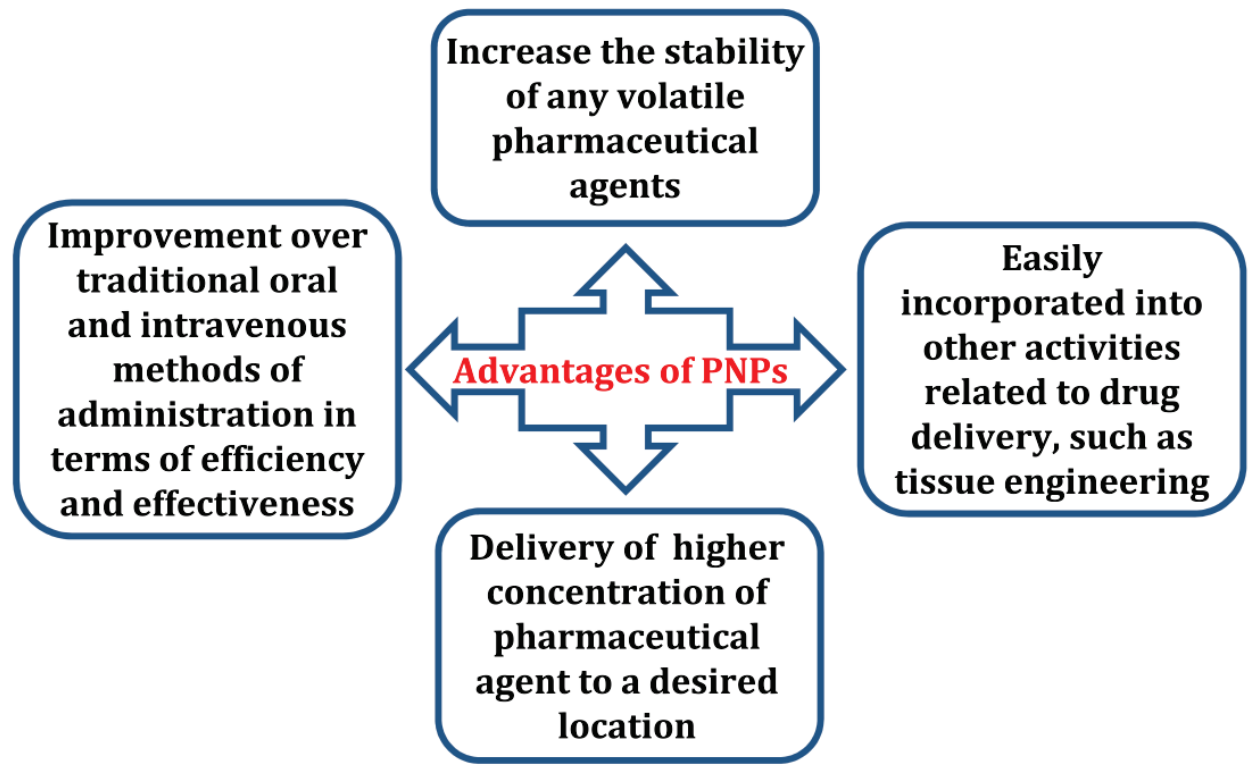

Scheme 1. Advantages of polymeric nanoparticles (PNPs)

\subsubsection{Methods for nanoparticle preparation}

The properties of PNPs have to be optimised in accordance with the particular application. In order to achieve the properties of interest, the mode of preparation plays a vital role. Thus, it is highly advantageous to have appropriate preparation techniques at hand in order to obtain PNPs with the desired properties for a particular application. Several methods have been developed with nanoparticle sizes generally ranging in the scale of 100-500 nm [100]. These techniques are classified according to whether the particle formation involves a polymerisation reaction or a direct arrangement of pre-formed polymers and a desolvatation of macromolecules [102-104]. The polymerisation methods can be further classified into emulsion and interfacial polymerisation, and there are two types of emulsion polymerisation - organic and aqueous - depending on the continuous phase. The availability of different synthetic approaches allows for considerable flexibility in the preparation and functionalisation strategy of multifunctional PNPs.

\subsubsection{Self-assembly}

Due to their unique chemical structures, amphiphilic (co-)polymers tend to self-assemble into nano-aggregates in aqueous solution $[105,106]$. To obtain PNPs containing active organic substances, a mixture of amphiphilic (co-)polymers and organic active substances is firstly dissolved in a "good" solvent and then quickly added to an excess amount of a "poor" solvent. Thus, 
the hydrophobic segments of the polymer tend to aggregate and encapsulate the organic substance in the core, while the hydrophilic polymer segments act as a shell to stabilise the PNPs. Moreover, upon conjugating the active organic substance to the hydrophobic ends or side chains of the amphiphilic ( $\mathrm{co}$-)polymer, the polymer-substance conjugates form nanoparticles with the organic active substance embedded and anchored in the polymeric matrix. Additionally, the polymer hydrophilic segments could be decorated with functional groups for further conjugation with specific targeting moieties to cater to versatile biological tasks.

\subsubsection{Polymerisation}

In a typical polymerisation method, the organic solvent, containing monomers and organic active substances, is uniformly dispersed into stable and small oil droplets in the presence of an emulsifier in an aqueous solution through ultrasonification. The polymerisation of monomers in oil droplets starts with the addition of initiators into the emulsion to yield organic nanoparticle dispersions (Figure 4). Further solvent evaporation results in well-dispersed PNPs. In this method, the organic substance can be either reactive or nonreactive to the monomers during polymerisation $[107,108]$.

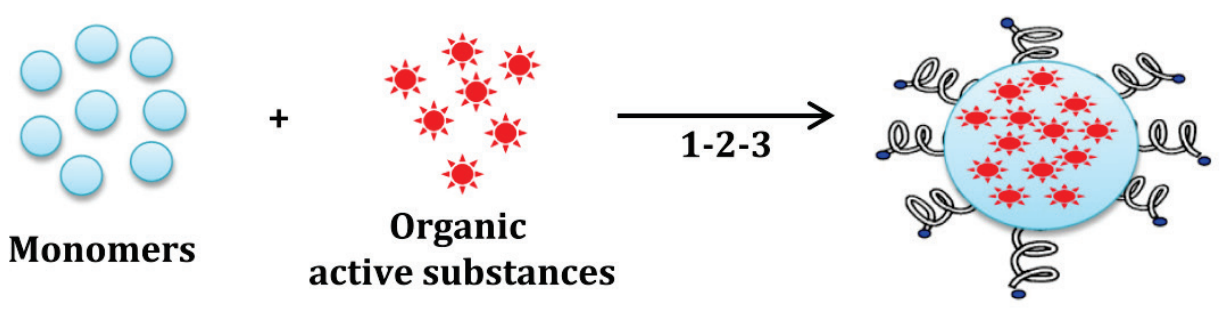

Figure 4. Schematic illustration of organic active substance-encapsulated PNPs prepared from in situ polymerisation: (1) Addition of monomers and organic substances in water containing an emulsifier; (2) dispersion with sonication;

(3) addition of an initiator to initiate the monomer polymerisation to yield organic substance-loaded PNPs

\subsubsection{Emulsification / solvent evaporation}

Emulsification-solvent evaporation involves two steps (Figure 5). The first step requires emulsification of the polymer solution into an aqueous phase. To do so, the polymer organic solution containing the dissolved drug is dispersed into nanodroplets, using a dispersing agent and high-energy homogenisation, in a non-solvent or suspension medium such as ethyl acetate. During the second step, polymer solvent is evaporated by increasing the temperature under pressure or by continuous stirring, inducing polymer precipitation in 
the form of nanospheres [109]. When the solvent is added into the emulsifier-containing aqueous solution under ultrasonication or vigorous stirring, small organic droplets are stabilised by the emulsifier to generate a homogeneous oil-in-water emulsion. After organic solvent evaporation, a stable suspension of PNPs in water is obtained and the nanoparticles surfaces can be used for further functionalisation. However, this method can only be applied to liposoluble drugs, and limitations are imposed by scale-up of the high energy requirements in homogenisation. Frequently used polymers in this method are PLA, PLGA, PCL, and PHB. Drugs or model drugs encapsulated in this way include: albumin, texanus taxoid, loperamide, pranziquantel, cyclosporin $\mathrm{A}$, nucleic acid and indomethacin.

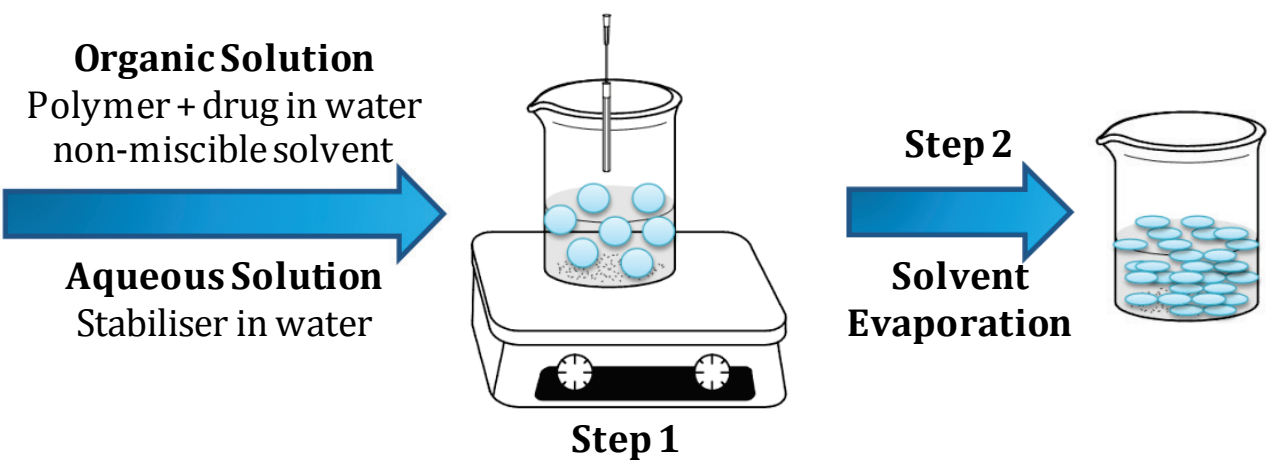

Figure 5. Schematic representation of the emulsification-evaporation technique

\subsubsection{Nanoprecipitation}

Nanoprecipitation is also known as the solvent displacement method. It differs from emulsion in organic solvents since it involves the precipitation of a pre-formed polymer from an organic solution and the diffusion of the organic solvent in an aqueous medium, in the presence or absence of a surfactant [109]. Using PLA as an example, PLA is dissolved in a water-miscible solvent of intermediate polarity and this phase is subsequently injected into a stirred aqueous solution containing a stabiliser surfactant. Polymer deposition on the interface between the water and the organic solvent, caused by fast diffusion of the solvent, leads to the instantaneous formation of a colloidal suspension [110]. To facilitate the formation of colloidal polymer particles during the first step of the procedure, phase separation is performed with a totally water-miscible solvent that is also a non-solvent of the polymer [111]. The solvent displacement technique allows for the preparation of nanocapsules when a small volume of non-toxic oil is incorporated into the organic phase. Considering the oil-based central cavities of the nanocapsules, high loading efficiencies are generally reported for lipophilic drugs when nanocapsules are 
prepared. Furthermore, hydrophilic segments of the polymeric matrix orient into the aqueous phase and facilitate further functionalisation of the PNPs. However, the usefulness of this simple technique is limited to water-miscible solvents, in which the diffusion rate is enough to produce spontaneous emulsification [110]. Moreover, even though some water-miscible solvents produce certain instability when mixed in water, spontaneous emulsification is not observed if the coalescence rate of the droplets is sufficiently high. Finally, this method is only practically applicable to lipophilic drugs because of the miscibility of the solvent with the aqueous phase, therefore it is not an efficient means to encapsulate water-soluble drugs. This method has been applied using various polymeric materials such as PLGA, PLA, PCL, and poly(methyl vinyl ether-comaleic anhydride) [112-115]. It has been well adapted for the incorporation of cyclosporin A, with entrapment efficiencies as high as $98 \%$ reported [116]. It can also be used for the preparation of highly loaded nanoparticulate systems based on amphiphilic h-cyclodextrins to facilitate the parenteral administration of the poorly soluble antifungal drugs Bifonazole and Clotrimazole.

\subsubsection{Salting-out}

Salting-out is based on the separation of a water miscible solvent from aqueous solution via a salting-out effect. The salting-out procedure can be considered as a modification of the nanoprecipitation process. The polymer and drug are initially dissolved in a solvent such as acetone, which is subsequently emulsified into an aqueous gel containing the salting-out agent (electrolytes such as magnesium chloride, calcium chloride, and magnesium acetate, or non-electrolytes such as sucrose) and a colloidal stabiliser such as poly(vinylpyrrolidone) (PVP) or hydroxyethylcellulose. The selection of the salting-out agent is important because it can play an important role in the encapsulation efficiency of the drug (Table 3). Stirring causes the dispersion of the solvent as irregular sized globules in equilibrium with the continuous phase, and the stabiliser is absorbed on the larger interface; further homogenisation results in smaller globules. The oil-in-water emulsion is diluted with a sufficient volume of water or aqueous solution to enhance the diffusion of acetone into the aqueous phase, thus inducing the formation of nanospheres. The addition of water and the heating step destabilises the equilibrium and causes the diffusion of the organic solvent to the external surface. During the transport of the solute, PNPs are produced with sizes ranging from $100-200 \mathrm{~nm}$. A heating step also encourages the production of a final suspension free of organic solvent which is more uniform in size. Both the solvent and the salting-out agent are then eliminated by cross-flow filtration [96]. To remove the non-encapsulated drug, the PNP suspension is generally filtered and ultracentrifuged followed by re-suspension in an adequate volume of water. The salting-out process has been used in the preparation of biodegradable PNPs with high efficiency and is easily scaled up. The main 
advantage of salting-out is that it minimises stress to the protein encapsulants [57]. It does not require an increase of temperature and therefore may be useful when heat sensitive substances have to be processed [117]. Moreover, the amount of drug encapsulated into the PNPs can be modulated by varying various parameters such as drug concentration, rate, temperature, and the nature of the polymer ligands. Some studies have suggested that drug loading can also be increased by varying the $\mathrm{pH}$ value of the solution $[118,119]$. The greatest disadvantages are exclusive application to lipophilic drugs and the extensive nanoparticle washing steps [120].

Table 3. Properties, advantages and disadvantages of some non-electrolyte salting-out agents and colloidal stabilisers

\begin{tabular}{ccc}
\hline $\begin{array}{c}\text { Salting-out agents and } \\
\text { colloidal stabilisers }\end{array}$ & Properties & Advantages/disadvantages \\
\hline Chitosan & $\begin{array}{c}\text { Natural, cationic, } \\
\text { hydrophilic, linear, } \\
\text { biodegradable }\end{array}$ & $\begin{array}{c}\text { Can be used in non-viral gene } \\
\text { delivery }\end{array}$ \\
\hline Dextran & $\begin{array}{c}\text { Natural, branched, } \\
\text { hydrophilic, } \\
\text { biocompatible }\end{array}$ & $\begin{array}{c}\text { Permits the anchoring of } \\
\text { biovectors and drugs when } \\
\text { functionalised with amino } \\
\text { groups }\end{array}$ \\
\hline Sucrose & Natural & $\begin{array}{c}\text { Improves the stability of } \\
\text { proteins during lyophilisation }\end{array}$ \\
\hline PEG & $\begin{array}{c}\text { Remains stable in high ionic } \\
\text { strength solutions with varying } \\
\text { pH values }\end{array}$ \\
& $\begin{array}{c}\text { Sydrophilic, linear, } \\
\text { biocompatible }\end{array}$ & $\begin{array}{c}\text { Enhances blood circulation time } \\
\text { (a few hours) }\end{array}$ \\
PVP & $\begin{array}{c}\text { Synthermits functionalisation } \\
\text { hydrophilic }\end{array}$ & $\begin{array}{c}\text { Forms covalent bonds with } \\
\text { drugs containing nucleophilic } \\
\text { functional groups }\end{array}$ \\
\hline
\end{tabular}




\subsubsection{Examples of nanoparticles obtained from biodegradable synthetic and natural polymers}

Chitosan-based nanoparticles have been widely developed to encapsulate proteins such as bovine serum albumin, tetanus and diphtheria toxoid vaccines, anticancer agents, insulin, and nucleic acids [121]. Chitosan enhances the absorption of peptides such as insulin and calcitonin across the nasal ephithelium. The methods proposed to prepare chitosan-based nanoparticles are based on the spontaneous formation of complexes between chitosan and polyanions or the gelation of a chitosan solution dispersed in an oil emulsion. The nanoparticles obtained by formation of the spontaneous complex have smaller diameters (200-500 nm).

Collagen has been widely used as a biomaterial for years due to its promising biocompatibility, low antigenicity and biodegradability [122]. Although collagen forms hydrogels without the use of chemical crosslinking, nanoparticle preparation needs additional chemical treatments due to weak mechanical strength. For instance, collagen nanoparticles are often prepared by electrostatic interactions with sodium sulfate employed as a desolvating agent [123]. A recent study reported on the preparation of collagen-based nanoparticles $(340 \mathrm{~nm}$ ) with methods using lipid vesicle cages, which allow for the control of both the particle dimensions and the gelling environment during the collagen polymerisation [123]. Due to the ease of particle size control, their large surface area, high adsorption capacity and dispersion ability in water, collagen-based nanoparticles can be applied for the sustained release of various drugs.

Gelatin solutions undergo a coil-triple helix transition followed by aggregation of the helices, enabling the formation of nanoparticles. Moreover, the high number of functional groups on the polymer backbone can be used for chemical modification such as for crosslinking and the addition of ligands. Thus, gelatin is a much used biopolymer in the production of nanoparticles to be used as delivery carriers. A number of methods have been reported to prepare gelatin-based nanoparticles including desolvation (a thermodynamically driven self-assembly process), emulsion and crosslinking with poly(ethylenimine) and glutaraldehyde, nanoprecipitation, coacervation, and the grafting of hydrophobic anhydrides to the amino groups of primitive gelatin to form self-assembled micelles [109,124-126]. Emulsified gelatin droplets can also be hardened by cooling the emulsion below the gelation point in an ice bath, resulting in gelatin-based gelled nanodroplets which can be subsequently cross-linked with formaldehyde [127]. The particle sizes range from between $100-600 \mathrm{~nm}$ with a mean of $280 \mathrm{~nm}$, and crosslinking can significantly increase the particle size. This technique is useful for heat sensitive drugs; however, a number of drugs can be undesirably covalently bound to the gelatin during the formaldehyde treatment. Furthermore, a 
significant disadvantage of the crosslinking agent relates to its toxicity, and this point must be carefully considered.

The PLGA-based nanoparticulate system is one of the most successful and interesting colloidal systems. Indeed, PLGA nanoparticles protect the therapeutic agents, increase their stability and can be used for the controlled delivery of therapeutic molecules with improved pharmacokinetic and pharmacodynamic profiles. However, PLGA nanoparticles suffer from a significant limitation due to their high level of opsonization by the reticuloendothelial system (RES) [128]. To address this negative aspect, several methods and procedures have been utilised for the surface modification of PLGA nanoparticles in order to produce PLGA-based nanoparticulate systems which are not readily recognised by RES. This goal has been achieved by coating the surface of the nanoparticles with more hydrophilic agents to cover the hydrophobic surface and to provide stealth nanoparticles. Moreover, in some applications such as bone tissue engineering, there is a need for a resorbable polymeric systems with sufficient strength and toughness. It is most likely that a single phase system would not possess all the necessary material characteristics, and hence modifications of the polymer matrix may be needed to modulate the properties of the composite and generate the required bioactive material. However, separation at the composite filler-polymer interface suggests that bonding between all of the components of a multi-phase system is required to obtain a mechanically and thermally stable material. In this context, the development of PLGA-hydroxyapatite (nHAP) nanoparticles functionalised by collagen is a promising approach [129]. This synthesis is carried out in several steps. In the first step, the ring-opening polymerisation of D,L-lactide and glycolide monomers is initiated by hydroxyapatite nanoparticles. Thereafter, the polymerisation product is activated for collagen attachment (Figure 6). 


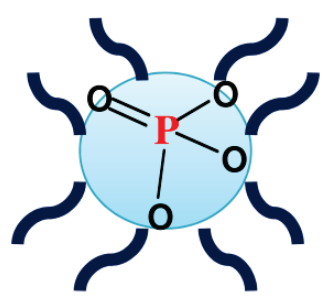

PLGA-nHAP
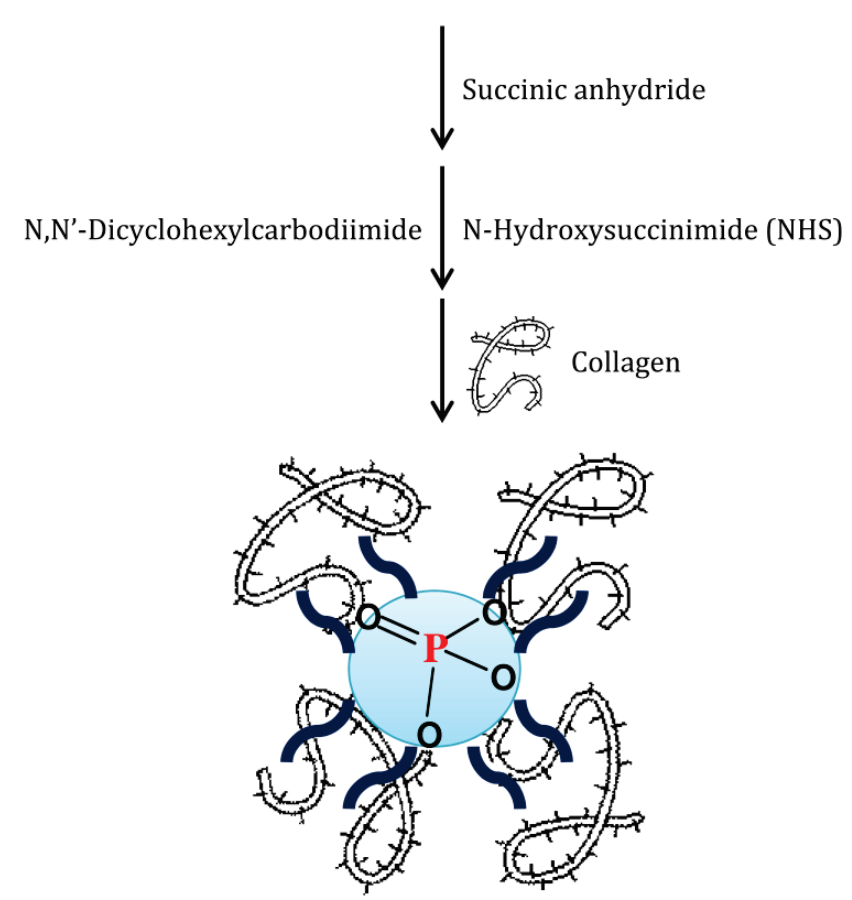

Col-PLGA-nHAP

Figure 6. Schematic representation of the synthesis of collagen-PLGA-nHAP nanoparticles: formation of PLGA-nHAP system followed by activation and attachment of collagen [129]

\subsection{SURFACE MODIFICATION}

Polymeric nanoparticles, nanofibres and nanofibrous scaffolds have been developed for a variety of applications in the medical field, such as for tissue engineering and for the delivery of bioactive molecules. However, while the biodegradability and bulk properties may match the needs of the intended application and generally motivates the polymer choice, the inert nature of synthetic biodegradable polymers may not facilitate the attachment of bioactive compounds and can lead to a lack of biological recognition. For 
instance, the surface chemical nature plays a vital role in tissue engineering because it impacts on the biological response [130]. To develop nanoparticles, nanofibres and nanofibrous scaffolds as useful nanobiomaterials, it is essential to tailor their surface properties and therefore a variety of approaches leading to high functionalities have been developed as post-treatments $[49,89,131,132]$. Consequently, surface-functionalised nanomaterials allow for coupling with a wide range of biological and therapeutic molecules that may be released in a timely and proper manner or that may provide bio-modulating or biomimetic microenvironments for cells and tissues [89].

\subsubsection{Surface modification of nanofibrous scaffolds}

In the biomaterial field, a variety of approaches have been proposed to change the polymer surface: introduction of polar groups by surface treatment, adsorption of biomolecules, and the covalent immobilisation of bioactive compounds [89,133-138]. In theory, any techniques used for the surface modification of polymeric biomaterials may be applied to polymeric nanofibres and nanofibrous scaffolds, as long as the process does not degrade the polymer nor changes its nano-features.

Simple physical adsorption is the most straightforward and convenient method to immobilise bioactive agents, such as enzymes, proteins, growth factors and drugs, onto a polymeric surface. In this technique, electrostatic interactions, hydrogen bonding, hydrophobic interactions, or van der Waals interactions are the driving forces leading to the surface adsorption. The efficiency of the adsorption may be enhanced by surface treatments that modify the hydrophilicity of the materials, such as plasma treatment or wet chemical methods [131]. Although it is the simplest approach to functionalisation, the control over the bioactive agent retention is limited, and conformational change and loss of bioactivity may take place when immobilising proteins [49,133]. However, non-covalent adsorption is sometimes desirable in drug delivery applications. For instance, postsurgical anti-adhesion barriers are biomaterials that physically separate the wound site from an adjacent organ or tissue, and concomitantly deliver infection preventing antibiotics. Consequently, a rapid drug release profile is highly desirable [89]. Another way to physically immobilise charged therapeutic molecules is the use of layer-by-layer assembly. In this method, polyanions and polycations are alternately deposited on a charged polymer surface, resulting in a multilayer coating in which the drug is encapsulated. This functionalisation technique is easy to implement and can be used with a wide compositional range of the coating layer. However, it is not applicable to uncharged drugs and the release profile is influenced by the thickness of the coating [89].

In contrast with physical adsorption, covalent immobilisation provides a stable bond between the bioactive compound and the polymer surface, and therefore 
provides a more efficient coating, extends the bioactive agent retention, and may prevent its metabolism [133]. Prior to the biomolecule covalent immobilisation, the generation of reactive functional groups on the polymer surface is necessary. This may be achieved by various wet chemical or plasma treatments. For instance, carboxylic acid and amine groups can be generated with oxygen, ammonia, or air plasma treatments [132]. They also may be generated on biodegradable polyesters through hydrolysis with sodium hydroxide and aminolysis with a diamine, respectively $[136,137,139,140]$. However, strong reaction conditions should be avoided to ensure maintenance of nano-feature and polymer. Subsequently, after activation of the functionalised polymer surface, the biomolecule is tethered directly or through the use of a linker molecule. A variety of conjugation techniques have been developed [133]. Covalent bonding and physical adsorption may also be combined. For instance, various methods have been considered to covalently attach heparin onto polymeric scaffolds for tissue engineering applications. Afterwards, growth factors can be bound to heparin with preservation of their stability and biological activity through the retention of their native conformation $[137,141]$. In another example, hydroxyapatite was coated onto nanofibrous biodegradable polyester (PLA or PCL) surfaces [131,139]. In a first step, the nanofibres surface was activated in an alkaline solution in order to generate carboxylic acid groups. In a second step, the nanofibrous material was dipped in $\mathrm{Ca}$ and P-rich solutions and finally, immersed in simulated body fluid. The mineralization occurs through the carboxylic acid groups that chelate calcium ions and initiate the mineral nucleation. The coating can be tailored by varying the incubation time and the simulated body fluid composition. In the end, the mineralised polymer exhibited higher osteoblastic responses (cells adhesion and growth) with better expression of the bone extracellular matrix genes than those on unmodified nanofibres. These new nanofibrous materials have potential as bone regeneration membrane [139].

The cost and the control for the covalent immobilisation of biomolecules remains a critical step for controlling the cell response. Moreover, the covalent bonding may partially inactivate the bioactive compound. Therefore, the introduction of multi-functional groups is an easier way to change the charge or the chemical composition surface of a polymer, and therefore to tune the rearrangements of proteins that adsorb from the cell culture serum onto the polymer surface and to modulate the ultimate cell response [142,143]. With this in mind, surface graft polymerisation is a simple, effective, and versatile approach with a wide range of possibilities in terms of functionalisation and composition due to the vast choice of monomers available. Moreover, multifunctional groups can be introduced with a high density without modifying the polymer bulk properties. Surface graft polymerisation can be initiated by various techniques, such as plasma discharge, ultraviolet light, ozone oxidation, $\gamma$-rays, electron beams, and Cerium IV treatment [144]. Again, the processing conditions should be carefully controlled to ensure the polymer and 
nano-feature integrity is maintained. Furthermore, to confer surface hydrophilicity and provide functional groups for the subsequent immobilisation of bioactive compounds, surface graft polymerisation can also be used to covalently attach bioactive polymers, such as poly(sodium styrene sulfonate), which is known to influence protein adsorption and cell response $[138,145]$.

\subsubsection{Coupling strategies for the biofunctionalisation of nanoparticles}

The association of one or more biologically relevant molecules at the interface of nanoparticles defines a nanoparticle-bioconjugate with biological activity such as selective binding. Biomolecules of interest may include one or more of the following:

- Peptides, proteins, and antibodies

- Enzymes and ribozymes

- Oligonucleotides and aptamers

- Carbohydrates

- Lipids

- Drugs or other biologically active small molecules

The interest in these bioconjugate materials arises from the combination of nanoscale size with the nearly infinite diversity of physical properties and chemical functionality that can be obtained through organic chemistry. Indeed, PNPs can be designed to:

- Carry molecular cargo externally or internally

- Carry hydrophilic or hydrophobic cargo

- Release cargo gradually

- Exhibit "smart" physicochemical responses to environmental stimuli (e.g., $\mathrm{pH}$, thermal response)

- Evade the reticuloendothelial system and other immune responses

- Biodegrade

- Target different tissues or cell types

These different properties are tailored through the selection of the chemical composition of the PNPs. Bioconjugates of polymer and amphiphile nanoparticles are typically prepared to assist targeting, with antibody conjugates being particularly common. While there is no characteristic surface chemistry due to the diversity of materials, the introduction of carboxylic acid or amine groups into the polymer / amphiphile composition for bioconjugation is routine. Overall, the bioconjugation chemistry of PNPs is generally dictated by the functional groups associated with the material. 


\subsubsection{Methods of coupling}

Various coupling methods have been widely studied (Figure 7). Carbodiimide coupling is used to covalently link carboxylic acid to amine groups via formation of a "zero length" amide bond [146]. The key advantage of this procedure is that it involves no lengthy linker species, so that the hydrodynamic radius of the PNPs is minimised. The most common carbodiimide coupling strategy uses 1-ethyl-3-(dimethylaminopropyl) carbodiimide hydrochloride (EDC or EDAC) as the coupling agent, which has been applied for enzyme-to-PNP coupling with retention of up to $50-80 \%$ of the enzymatic activity, depending on the enzyme [147,148] (Figure 7-A). The efficiency of the coupling reaction can be increased by stabilising the $O$-acylisourea intermediate by formation of the succinimide ester using $N$-hydroxysuccinimide (NHS) or sulfo-NHS. Bis( $N$-hydroxysuccinimide) can also be used without a carbodiimide activation agent, allowing for the conjugation of two amine moieties thanks to the two NHS ester groups [149]. When PNPs bear hydroxyl groups on the surface, the activated species may be directly coupled via a net dehydration reaction leading to ester linkages [150]. Maleimide may be used to conjugate primary amines to thiol groups as illustrated in Figure 7-B [151]. The most commonly used maleimide-derived coupling reagent is sulfosuccinimidyl-4-(maleimidomethyl)cyclohexane-1-carboxylate (sulfo-SMCC). Maleimide coupling has been widely used to conjugate biomolecules such as DNA, herceptin and proteins [152-154].

Another common route for bioconjugation is the $\mathrm{Cu}(\mathrm{I})$-catalysed alkyne-azide cycloaddition reaction, also known as "CuAAC" or "click chemistry", which involves the coupling of an alkyne group to an azide moiety resulting in a 1,2,3-triazole ring as the strong covalent bond between the PNPs and the biofunctional agents (Figure 7-C). This process has been demonstrated to be highly versatile since either alkyne or azide moieties can be expressed on the biofunctional agent, suitable for conjugation of a variety of species including small molecules. Combined with the variety of ligand head groups available for nanoparticle-ligand bond formation, this procedure has a lot of potential as a coupling approach for bioconjugation. Furthermore, the one-step click process has been shown to give the possibility of introducing multiple functionalities onto PNPs [155].

Finally, charged PNPs may be coupled either with oppositely charged biological and polymeric species, or indeed to different oppositely charged nanoparticles (Figure 7-D) [156,157]. Some obvious examples of biological application are the coupling of negatively charged DNA or liposomes to positively charged PNPs [158,159]. Moreover with careful tuning of the $\mathrm{pH}$, it is possible to couple a variety of proteins, which can be cationic, anionic or neutral [160]. 


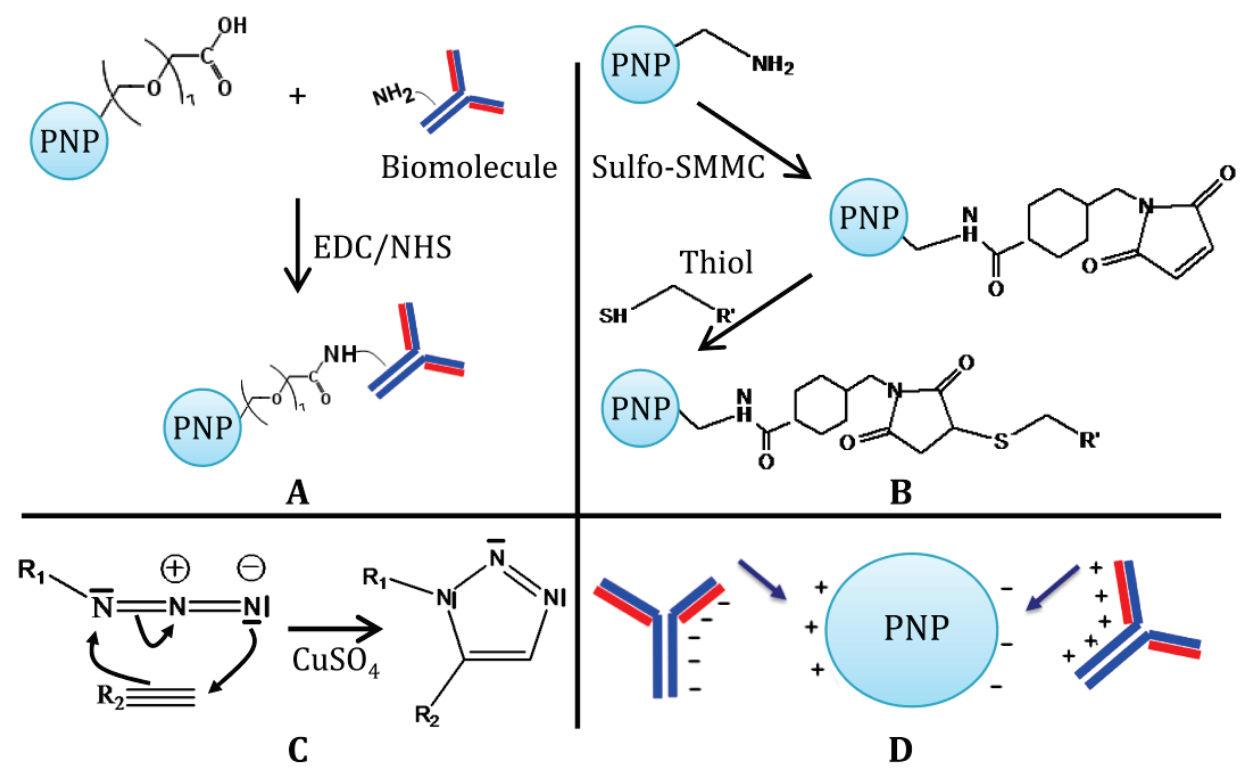

Figure 7. Schematic representation of various coupling methods: A) carbodiimide coupling used to covalently link PNP bearing carboxylic acid groups to amine moietiesbearing antibodies, B) Maleimide coupling of amines with thiol groups using sulfoSMMC as the linker, C) Schematic of Cu-mediated alkyne-azide cycloaddition ("click reaction"), D) Ionic coupling between antibodies and PNPs

\subsubsection{Polymers as conjugating agents on PNPs}

Small molecules can be used as ligands to act as a sort of physical barrier preventing the nanoparticle cores from coming into contact with each other. This approach is associated with a very small increase in the nanoparticle hydrodynamic radius. However, care must be taken not to make the molecular shell too thin as this leads to an insufficient steric barrier, resulting in reduced nanoparticle stability and aggregation [112]. By contrast, polymers also make excellent ligands to surround nanoparticles and act as a substantial physical barrier, which leads to a higher hydrodynamic radius in comparison to using small ligands [128]. This effect is desirable for in vivo applications requiring a long circulation time, but disadvantageous if rapid diffusion to the extravascular space is required; essentially, size is a very important factor in the biodistribution of PNPs [161]. Indeed like small-molecule stabilising agents, the concentration of polymeric stabilisers may be used to control the nanoparticle core morphology $[112,159]$.

There are many suitable polymeric ligands that enable water solubility, most of which are based on PEG and common carbohydrates such as starch, dextran and chitosan [102,103,121]. PEG is especially suitable for nanosystems 
requiring long circulation times in blood, as it reduces the degree of opsonisation and provides excellent long-term stability in high salt concentrations and pH extremes [162]. Moreover, PEG end chains can be modified to provide chemical functionality or ionic stabilisation, and allow for the selective attachment to nanoparticle surfaces and subsequent biofunctionalisation (Table 4) [156]. Therefore, PNPs decorated with different ligands could be used for therapeutical purposes. For instance, appropriate functionalisation of PNPs with targeting moieties could enable selective recognition and interaction with specific cancer cells. Indeed, the proper orientation of the targeting moiety can promote the antigen binding and the subsequent spontaneous cell internalisation.

Finally, nanoparticle surface charge can also have an important influence on their interaction with cells and on their uptake. Positively charged nanoparticles seem to allow for a higher extent of internalisation, due to the ionic interactions established between the positively charged nanoparticles and the negatively charged cell membranes [159]. Moreover, positively charged nanoparticles seem to be able to escape from lysosomes after being internalised and exhibit perinuclear localisation, whereas the negatively and neutrally charged nanoparticles prefer to colocalise with lysosomes. Nanoparticle surface charges can be modulated through functionalisation with polymeric ligands to facilitate a higher degree of internalisation. For instance, PLGA nanoparticles have negative charges which can be shifted to neutral or positive charges by surface modification with PEG or chitosan, respectively $[12,121]$.

Table 4. Examples of PEG derivative ligands used for chemical functionalisation of PNPs

\begin{tabular}{ccc}
\hline $\begin{array}{c}\text { PEG derivatives used as } \\
\text { ligands }\end{array}$ & Chemical structure & Functionalisation \\
\hline $\begin{array}{c}\text { PEG } \\
\text { bis(carboxymethyl)ether }\end{array}$ & $\begin{array}{c}\text { Carbodiimide coupling } \\
\text { Ionic PNPs }\end{array}$ \\
\hline Chiol-PEG-carboxylic ether & $\begin{array}{c}\text { Carbodiimide coupling } \\
\text { Ionic PNPs }\end{array}$ \\
\hline PEG bis(amine) & PEG bis(azide) & $\begin{array}{c}\text { Carbodiimide coupling } \\
\text { Ionic PNPs }\end{array}$ \\
\hline
\end{tabular}




\subsection{CONCLUSION}

After a presentation of the biodegradable polymers used in medical applications, this chapter gave an overview of the principal approaches that can generate nanofibres and nanofibrous scaffolds as well as nanoparticles. The choice of the method and the processing parameters are crucial for the control of the nanofibrous and nanoparticle architecture and size as well as their properties. Post-treatments and conjugations are often applied to modify the surface chemistry leading to functionalised materials with biological cues. Due to the annual growing of the global nanomaterial market, research and development at the material scale is still challenging for the production of new nanomaterials that could be used as biomimicking scaffolds and bioactive nanocarriers.

\section{REFERENCES}

1. E. Campos, R. Cordeiro, A.C. Santos, C. Matos, M.H. Gil. Colloids Surf. B Biointerfaces 88 (2011) 477-482.

2. Transparency Market Research, Nanomaterials Market - Global Industry Analysis, Size, Share, Growth, Trends and Forecast 2014 - 2020, 2014.

3. $\quad$ L.S. Nair, C.T. Laurencin. Prog. Polym. Sci. 32 (2007) 762-798.

4. S. Naahidi, M. Jafari, F. Edalat, K. Raymond, A. Khademhosseini, P. Chen. J. Control Release 166 (2013) 182-194.

5. R. Vasita, D. S. Katti. Int. J. Nanomedicine 1 (2006) 15-30.

6. A. Kumari, S.K. Yadav, S.C. Yadav. Colloids Surf. B Biointerfaces 75 (2010) 1-18

7. A. Shukla, R. Tiwari, Intelligent Medical Technologies and Biomedical Engineering: Tools and Applications, IGI Global, Hershey, USA, 2010, p. 376.

8. S. Deb, in Biomaterials Fabrication and Processing Handbook, P.K. Chu, X. Liu, (Eds.), CRC Press, Boca-Raton, USA, 2008, p. 457-481.

9. D.M. Yoon, J.P. Fisher, in Biomedical Materials, R. Narayan (Ed.), Springer, New York, USA, 2009, p. 415-441.

10. S. Dumitriu, Polymeric Biomaterials, $2^{\text {nd }}$ Edition, Revised and Expanded, Marcel Dekker, New York, USA, 2002, p. 1168.

11. Q.-Z. Chen, O. Bretcanu, A.R. Boccaccini, in Biomaterials Fabrication and Processing Handbook, P.K. Chu, X. Liu (Eds.), CRC Press, Boca-Raton, USA, 2008, p. 3-43.

12. F. Danhier, E. Ansorena, J.M. Silva, R. Coco, A. Le Breton, V. Préat. J. Control Release 161 (2012) 505-522.

13. U. Edlund, A.-C. Albertsson, in Degradable Aliphatic Polyesters, Advances in Polymer Science, vol. 157, A.C. Albertsson (Ed.), Springer-Verlag, Berlin-Heildeberg, Germany, 2002, p. 67-112.

14. B.D. Ulery, L.S. Nair, C.T. Laurencin. J. Polym. Sci. Part B: Polym. Phys. 49 (2011) 832-864.

15. N. Duzgunes, Methods in Enzymology. Vol. 509. Nanomedicine: Infectious Diseases, Immunotherapy, Diagnostics, Antifibrotics, Toxicology and Gene Medicine, Academic Press, San Diego, USA, 2012, p. 470. 
16. M.J. McClure, S.A. Sell, D.G. Simpson, B.H. Walpoth, G.L. Bowlin. Acta Biomater. 6 (2010) 2422-2433.

17. W.-J. Li, R. Tuli, C. Okafor, A. Derfoul, K.G. Danielson, D.J. Hall, R.S. Tuan, Biomaterials 26 (2005) 599-609.

18. J. Ramier, T. Bouderlique, O. Stoilova, N. Manolova, I. Rashkov, V. Langlois, E. Renard, P. Albanese, D. Grande. Mater. Sci. Eng. C Mater. Biol. Appl. 38 (2014) 161-169.

19. F.S. Poletto, L.A. Fiel, B. Donida, M.I. Ré, S.S. Guterres, A.R. Pohlmann. Colloids Surf. A Physicochem. Eng. Aspects 324 (2008) 105-112.

20. C. Peña, T. Castillo, A. García, M. Millán, D. Segura. Microbial Biotech. 7 (2014) 278-293.

21. M.C. Hacker, A.G. Mikos, in Principles of Regenerative Medicine, A. Atala, R. Lanza, J.A. Thomson, A. Nerem (Eds.), Elsevier, Burlington, USA, 2008, p. 604-635

22. W.-C. Lee, I.-M. Chu. J. Biomed. Mater. Res. Part B: Appl. Biomater. 84B (2008) 138-146.

23. X. Jiang, H. Xin, J. Gu, X. Xu, W. Xia, S. Chen, Y. Xie, L. Chen, Y. Chen, X. Sha, X. Fang. Biomaterials 34 (2013) 1739-1746.

24. R. James, M. Deng, S.G. Kumbar, C.T. Laurencin, in Natural and Synthetic Biomedical Polymers, S.G. Kumbar, C.T. Laurencin, M. Deng (Eds.), Elsevier, Burlington, USA, 2014, p. 193-205.

25. A.K. Andrianov, R. Langer, in Polyphosphazenes for Biomedical Applications, A.K. Andrianov (Ed.), John Wiley \& Sons, Hoboken, USA, 2009, p. 3-14.

26. C. Diaz, M.L. Valenzuela, in Polyphosphazenes for Biomedical Applications, A.K. Andrianov (Ed.), John Wiley \& Sons, Hoboken, USA, 2009, p. 297-324.

27. G. Rohman, in Biomaterials, V. Migonney (Ed.), John Wiley \& Sons, Hoboken, USA, 2014, p. 27-81.

28. Y. Wen, J.K. Oh. Macromol. Rapid Commun. 35 (2014) 1819-1832.

29. K.Y. Lee, L. Jeong, Y.O. Kang, S.J. Lee, W.H. Park. Adv. Drug. Deliv. Rev. 61 (2009) 1020-1032.

30. E.L. Monzack, K. J. Rodriguez, C.M. McCoy, X. Gu, K.S. Masters, in Biomaterials for Tissue Engineering Applications - A Review of the Past and Future Trends, J.A. Burdick, R.L. Mauck (Eds.), Springer-Verlag, Wien, Germany, 2011, p. 209-241.

31. W. Paul, C.P. Sharma, in Degradation Rate of Bioresorbable Materials Prediction and Evaluation, F. Buchanan (Ed.), CRC Press, Boca-Raton, USA, 2008, p. 67-94.

32. C.A. Schütz, L. Juillerat-Jeanneret, P. Käuper, C. Wandrey, Biomacromolecules 12 (2011) 4153-4161.

33. H.A. Patel, J.K. Patel. Int. J. Pharm. Sci. Rev. Res. 4 (2010) 37-41.

34. J. Maia, M.B. Evangelista, H. Gil, L. Ferreira, in Carbohydrates Applications in Medicine, M.H. Gil (Ed.), Research Signpost, Kerala, India, 2014, p. 31-53.

35. S. Zhou, H. Dou, Z. Zhang, K. Sun, Y. Jin, T. Dai, G. Zhou, Z. Shen. Polym. Chem. 4 (2013) 4103-4112.

36. R.A. Scott, A. Panitch. Wiley Interdiscip. Rev. Nanomed. Nanobiotechnol. 5 (2013) 388-398.

37. E.J. Oh, K. Park, K.S. Kim, J. Kim, J.-A. Yang, J.-H. Kong, M.Y. Lee, A.S. Hoffman, S.K. Hahn. J. Control. Release 141 (2010) 2-12.

38. E.M. Bueno, J. Glowacki. Nat. Rev. Rheumatol. 5 (2009) 685-697. 
39. K.Y. Choi, H. Chung, K.H. Min, H.Y. Yoon, K. Kim, J.H. Park, I.C. Kwon, S.Y. Jeong. Biomaterials 31 (2010) 106-114.

40. D.B. Khadka, D.T. Haynie. Nanomedicine: NBM 8 (2012) 1242-1262.

41. I. Vroman, L. Tighzert. Materials 2 (2009) 307-344.

42. X. Zhang, M.R. Reagan, D.L. Kaplan. Adv. Drug. Deliv. Rev. 61 (2009) 988-1006.

43. Transparency Market Research, Nano-Fiber Cell Scaffold Market - Global Industry Analysis, Size, Share, Growth, Trends and Forecast, 2012 - 2018, 2014.

44. B. Ma, J. Xie, J. Jiang, F.D. Shuler, D.E. Bartlett. Nanomedicine (Lond) 8 (2013) 33.

45. M. Prabaharan, R. Jayakumar, S.V. Nair, in Biomedical Applications of Polymeric Nanofibers, Advances in Polymer Science, vol. 246, R. Jayakumar, S.V. Nair (Eds.), Springer-Verlag, Berlin-Heildeberg, Germany, 2012, p. 241-262.

46. N. Bhardwaj, S.C. Kundu. Biotechnol. Adv. 28 (2010) 325-347.

47. A.J. Meinel, O. Germershaus, T. Luhmann, H.P. Merkle, L. Meinel. Eur. J. Pharm. Biopharm. 81 (2012) 1-13.

48. J.M. Holzwarth, P.X. Ma. Biomaterials 32 (2011) 9622-9629.

49. Z. Zhang, J. Hu, P.X. Ma. Adv. Drug. Deliv. Rev. 64 (2012) 1129-1141.

50. X. Liu, P.X. Ma. Biomaterials 30 (2009) 4094-4103.

51. J. Mey, G. Brook, D. Hodde, A. Kriebel, in Biomedical Applications of Polymeric Nanofibers, Advances in Polymer Science, vol. 246, R. Jayakumar, S.V. Nair (Eds.), Springer-Verlag, Berlin-Heildeberg, Germany, 2012, p. 131-170.

52. S.J. Lee, J.J. Yoo, in Handbook of Intelligent Scaffold for Tissue Engineering and Regenerative Medicine, G. Khang (Ed.), Pan Stanford Publishing, Singapore, 2012, p. 201-218.

53. N. Lavielle, A.-M. Popa, M. de Geus, A. Hébraud, G. Schlatter, L. Thöny-Meyer, R.M. Rossi. Eur. Polym. J. 49 (2013) 1331-1336,

54. P.S. Gungor-Ozkerim, T. Balkan, G.T. Kose, A. Sezai Sarac, F.N. Kok. J. Biomed. Mater. Res. Part A 102A (2014) 1897-1908.

55. L. Van der Schueren, B. De Schoenmaker, Ö.I. Kalaoglu, K. De Clerck. Eur. Polym. J. 47 (2011) 1256-1263.

56. X. Qin, D. Wu. J. Therm. Anal. Calorim. 107 (2012) 1007-1013.

57. J. Xie, X. Li, Y. Xia. Macromol. Rapid. Commun. 29 (2008) 1775-1792.

58. Z. Khatri, R. Nakashima, G. Mayakrishnan, K.-H. Lee, Y.-H. Park, K. Wei, I.-S. Kim. J. Mater. Sci. 48 (2013) 3659-3664.

59. R.K. Singh, K.D. Patel, J. Ho Lee, E.-J. Lee, J.-H. Kim, T.-H. Kim, H.-W. Kim. PloS One 9 (2014) e91584.

60. P.X. Ma, R. Zhang. J. Biomed. Mater. Res. 46 (1999) 60-72.

61. X.-T. Li, Y. Zhang, G.-Q. Chen. Biomaterials 29 (2008) 3720-3728.

62. J. Xie, X. Li, J. Lipner, C.N. Manning, A.G. Schwartz, S. Thomopoulos, Y. Xia, Nanoscale 2 (2010) 923-926.

63. A.O. Gee, B.M. Baker, A.M. Silverstein, G. Montero, J.L. Esterhai, R.L. Mauck. Cell Tissue Res. 347 (2012) 803-813.

64. S.H. Lim, H.-Q. Mao. Adv. Drug. Deliver. Rev. 61 (2009) 1084-1096.

65. W.-J. Li, J.A. Cooper Jr, R.L. Mauck, R.S. Tuan. Acta Biomater. 2 (2006) 377-385.

66. D. Han, K.C. Cheung. Polymers 3 (2011) 1684-1733.

67. V.J. Chen, P.X. Ma. Biomaterials 27 (2006) 3708-3715.

68. K. Zhang, A. Yin, C. Huang, C. Wang, X. Mo, S.S. Al-Deyab, M. El-Newehy. Polym. Degrad. Stabil. 96 (2011) 2266-2275. 
69. W. Ji, F. Yang, H. Seyednejad, Z. Chen, W.E. Hennink, J.M. Anderson, J.J.J.P. van den Beucken, J.A. Jansen. Biomaterials 33 (2012) 6604-6614.

70. H. Rodriguez-Tobias, G. Morales, A. Ledezma, J. Romero, D. Grande. J. Mater. Sci. 49 (2014) 8373-8385.

71. A. Suslu, A.Z. Albayrak, A.S. Urkmez, E. Bayir, U. Cocen, J. Mater. Sci.: Mater. Med. 25 (2014) 2677-2689.

72. L.S. Nair, S. Bhattacharyya, J.D. Bender, Y.E. Greish, P.W. Brown, H.R. Allcock, C.T. Laurencin. Biomacromolecules 5 (2004) 2212-2220.

73. M.Z. Elsabee, H.F. Naguib, R. Elsayed Morsi. Mater. Sci. Eng. C Mater. Biol. Appl. 32 (2012) 1711-1726.

74. C.A. Bonino, K. Efimenko, S. In Jeong, M.D. Krebs, E. Alsberg, S.A. Khan. Small 8 (2012) 1928-1936.

75. F. Cengiz-Çalloğlu. e-Polymers 14(2014) 5-13.

76. I. Chul Um, D. Fang, B.S. Hsiao, A. Okamoto, B. Chu. Biomacromolecules 5 (2004) 1428-1436.

77. M. Dubský, S.A. Kubinová, J. Sirc, L. Voska, R. Zajícek, A. Zajícová, P. Lesný, A. Jirkovská, J. Michalek, M. Munzarová, V. Holan, E. Syková.

J. Mater. Sci.: Mater. Med. 23 (2012) 931-941.

78. L. He, B. Liu, G. Xipeng, G. Xie, S. Liao, D. Quan, D. Cai, J. Lu, S. Ramakrishna. Eur. Cell Mater. 18 (2009) 63-74.

79. J. Mao, S. Duan, A. Song, Q. Cai, X. Deng, X. Yang. Mater. Sci. Eng. C Mater. Biol. Appl. 32 (2012) 1407-1414.

80. E. Guadalupe, D. Ramos, N.B. Shelke, R. James, C. Gibney, S.G. Kumbar. J. Appl. Polym. Sci. 132 (2015) 41879.

81. J.D. Schiffman, C.L. Schauer. Biomacromolecules 8 (2007) 594-601.

82. E. Sachlos, J.T. Czernuszka. Eur. Cell Mater. 5 (2003) 29-40.

83. P.X. Ma. Mater. Today 7 (2004) 30-40.

84. F. Gelain, A. Horii, S. Zhang. Macromol. Biosci. 7 (2007) 544-551.

85. S. Agarwal, J.H. Wendorff, A. Greiner. Polymer 49 (2008) 5603-5621.

86. S. Srinivasan, R. Jayakumar, K.P. Chennazhi, E.J. Levorson, A.G. Mikos, S.V. Nair, in Biomedical Applications of Polymeric Nanofibers, Advances in Polymer Science, vol. 246, R. Jayakumar, S.V. Nair (Eds.), Springer-Verlag, BerlinHeildeberg, Germany, 2012, p. 1-20.

87. O. Ishii, M. Shin, T. Sueda, J.P. Vacanti. J. Thorac. Cardiovasc. Surg. 130 (2005) 1358-1363.

88. M. Zamani, M.P. Prabhakaran, S. Ramakrishna. Int. J. Nanomedicine 8 (2013) 2997-3017.

89. H.S. Yoo, T.G. Kim, T.G. Park. Adv. Drug Deliver. Rev. 61 (2009) 1033-1042.

90. X. Hu, S. Liu, G. Zhou, Y. Huang, Z. Xie, X. Jing. J. Control Release 185 (2014) $12-21$

91. L. Cruz, L.U. Soares Costa, T.D. Mezzalira, G.N.P. da Silveira, S.S. Guterres, A.R. Pohlmann. Int. J. Pharm. 313 (2006) 198-205.

92. E. Amaral, A. Grabe-Guimarães, H. Nogueira, G.L. Machado, G. Barratt, V. Mosqueira. Life Sci. 80 (2007) 1327-1334.

93. V.C. Furtado, P. Legrand, J.L Morgat, M. Vert, E. Mysiakine, R. Gref, J.P. Devissaguet, G. Barratt. Pharm. Res. 18 (2001) 1411-1419.

94. A.F. Ourique, A.R. Pohlmann, S.S. Guterres, R.C.R. Beck. Int. J. Pharm. 352 (2008) 1-4. 
95. S. Guinebretière, S. Brianc, H. Fessi, V.S. Teodorescu, M.G. Blanchin. Mater. Sci. Eng. C 21 (2002) 137-142.

96. C. Pinto, R.J. Neufeld, A.J. Ribeiro, F. Veiga. Nanomedicine NBM 2 (2006) 8-21.

97. N. Anton, J.P. Benoit, P. Saulnier. J. Control Release 128 (2008) 185-199.

98. M.V. Chaubal. Drug Discover. Today 9 (2004) 603-609.

99. K. Letchford, H. Burt. Eur. J. Pharm. Biopharm. 65 (2007) 259-269.

100. D. Quintanar, E. Allémann, H. Fessi, E. Doelker. Drug Dev. Ind. Pharm. 24 (1998) 1113-1128.

101. D. Moinard-Checot, Y. Chevalier, S. Briançon, H. Fessi, S. Guinebretière. J. Nanosci. Nanotechnol. 6 (2006) 2664-2681.

102. C. Vauthier, K. Bouchemal. Pharm. Res. 26 (2008) 1025-1058.

103. E. Fattal, C. Vauthier, in Encyclopedia of Pharmaceutical Technology, J. Swarbrick (Ed.), Marcel Dekker, New York, USA, 2002, p. 1864-1882.

104. L. Kai ,L. Bin. Chem. Soc. Rev. 43 (2014) 6570-6597.

105. M.J. Joralemon, S. Mcrae, T. Emrick. Chem. Commun. 46 (2010) 1377-1393.

106. G. Gaucher, R.H. Marchessault, J.C. Leroux. J. Control Release 143 (2010) 2-12.

107. J. Chen, F. Zeng, S. Wu, J. Su, Z. Tong. Small 5 (2009) 970-978.

108. S. Wang, G. Kim, Y.-E.K. Lee, H.J. Hah, M. Ethirajan, R.K. Pandey, R. Kopelman. ACS Nano 6 (2012) 6843-6851.

109. S. Hornig, T. Heinze, C.R. Becer, U.S. Schubert. J. Mater. Chem. 19 (2009) 3838-3840.

110. T. Govender, S. Stolnik, M.C. Garnett, L. Illum, S.S. Davis. J. Control Release 57 (1999) 171-185.

111. C. Vauthier, C. Dubernet, E. Fattal, P. Alphandary, P. Couvreur. Adv. Drug Deliv. Rev. 55 (2003) 519-548.

112. J.M. Barichello, M. Morishita, K.Takayama, T. Nagai. Drug Dev. Ind. Pharm. 6 (1999) 425-471.

113. F. Nemati, C. Dubernet, H. Fessi, A.C. Verdiere, M.F Poupon, F. Puisieux. Int. J. Pharm. 38 (1996) 237-246.

114. J. Molpeceres, M. Guzman, M.R. Aberturas, M. Chacon, L. Berges. J. Pharm. Sci. 85 (1996) 206-213.

115. J.M. Irache, M. Huici, M. Konecny, S. Espuelas, M.A. Campanero, P. Arbos, Molecules 10 (2005) 126-145.

116. E. Allemann, J.C. Leroux, R. Gurny. Adv. Drug Deliv. Rev. 34 (1998)171-189.

117. G. Lambert, E. Fattal, P. Couvreur. Adv. Drug Deliv. Rev. 47 (2001)99-112.

118. M. Gajendiran, S. Mohammed, J. Yousuf, V. Elangovan, S. Balasubramanian. J. Mater. Chem. B 2 (2014) 418-427.

119. F. Danhier, O. Feron, V. Préat. J. Control Release 148 (2010) 135-146.

120. P. Couvreur, C. Dubernet, F. Puisieux, Eur J Pharm Biopharm 41 (1995) 2-13

121. A. Rampino, M. Borgogna, P. Blasi, B. Bellich, A. Cesàro. Int. J. Pharm. 455 (2013) 219-228.

122. J.W. Stone, P.N. Sisco, E.C. Goldsmith, S.C. Baxter, C.J. Murphy. Nano Lett. 7 (2007) 116-119.

123. M. Papi, V. Palmieri, G. Maulucci, G. Arcovito, E. Greco, G. Quintiliani, M. Fraziano, M. De Spirito. J. Nanopart. Res. 13 (2011) 6141-6147.

124. S. Azarmi, Y. Huang, H. Chen, S. McQuarrie, D. Abrams, W. Roa, W.H. Finlay, G.G. Miller, R. Löbenberg. J. Pharm. Pharm. Sci. 9 (2006) 124-132.

125. W.-T. Kuo, H.-Y. Huang, M.-J. Chou, M.-C. Wu, Y.-Y. Huang. J. Nanomater. 2011 (2011) 646538. 
126. E. Leo, A. Vandelli, M.R. Cameroni, F. Forni. Int. J. Pharm. 155 (1997) 75-82.

127. A.K. Sailaja, P. Amareshwar. J. Pharm. Res. 5 (2012) 1854-1856.

128. L. Shydar, L. Huang. BBA-Biomembranes 10 (2009) 2259-2266.

129. D. Bhuiyan, M.J. Jablonsky, I. Kolesov, J. Middleton, T.M. Wick, R. Tannenbaum. Acta. Biomater. 15 (2015) 181-190.

130. D.G. Castner, B.D. Ratner. Surf. Sci. 500 (2002) 28-60.

131. V. Beachley, X. Wen. Prog. Polym. Sci. 35 (2010) 868-892.

132. H. Chen, R. Truckenmüller, C. Van Blitterswijk, L. Moroni, in Nanomaterials in Tissue Engineering: Fabrication and Applications, A.K. Gaharwar, S. Sant, M.J. Hancock, S.A. Hacking (Eds.), Woodhead Publishing Limited, Cambridge, United Kingdom, 2013, p. 158-183.

133. J.M. Goddard, J.H. Hotchkiss. Prog. Polym. Sci. 32 (2007) 698-725.

134. G. Chan, D.J. Mooney. Trends Biotechnol. 59 (2008) 382-392.

135. J.K. Tessmar, A.M. Göpferich. Adv. Drug Deliver. Rev. 59 (2007) 274-291.

136. M.-C. Serrano, R. Pagani, M. Vallet-Regí, J. Peña, J.-V. Comas, M.T. Portolés. Acta Biomater. 5 (2009) 2045-2053.

137. G. Rohman, S.C. Baker, N.R. Cameron, J.J. Southgate. Mater. Chem. 19 (2009) 9265-9273.

138. S. Huot, G. Rohman, M. Riffault, A. Pinzano, L. Grossin, V. Migonney. Bio-Med. Mater. Eng. 23 (2013) 281-288.

139. J.-H. Jang, O. Castano, H.-W. Kim. Adv. Drug Deliver. Rev. 61 (2009) 1065-1083.

140. D.R. Nisbet, L.M. Yu, T. Zahir, J.S. Forsythe, M.S. Shoichet. J. Biomater. Sci. Polym. Ed. 19 (2008) 623-634.

141. C.L. Casper, W. Yang, M.C. Farach-Carson, J.F. Rabolt. Biomacromolecules 8 (2007) 1116-1123.

142. S.G. Eskin, T.A. Horbett, L.V. McIntire, R.N. Mitchell, B.D. Ratner, F.J. Schoen, A. Yee, in Biomaterials Science: An introduction to materials in medicine, B.D. Ratner, A.S. Hoffman, F.J. Schoen, J.E. Lemons (Eds.), Elsevier Academic Press, San Diego, USA, 2004, p. 237-291.

143. K. Park, Y.M. Ju, J.S. Son, K.D. Ahn, D.K. Han. J. Biomater. Sci. Polym. Ed. 18 (2007) 369-382.

144. Z. Ma, Z. Mao, C. Gao. Colloids Surf. B Biointerfaces 60 (2007) 137-157.

145. N. Djaker, S. Brustlein, G. Rohman, S. Huot, M. Lamy de la Chapelle, V. Migonney. Biomed. Opt. Express 5 (2014) 149-157.

146. X.H. Gao, Y.Y. Cui, R.M. Levenson, L.W.K. Chung, S.M. Nie. Nat. Biotechnol. 22 (2004) 969-976.

147. M. Koneracka, P. Kopcansky, M. Antalik, M. Timko, C.N. Ramchand, D. Lobo, R.V. Mehta, R.V. Upadhyay. J. Magn. Magn. Mater. 201 (1999) 427-430.

148. L.D. Chen, J. Liu, X.F. Yu, M. He, X.F. Pei, Z.Y. Tang. Biomaterials 29 (2008) 4170-4176.

149. P.C. Lin, P.H. Chou, S.H. Chen, H.K. Liao, K.Y. Wang, Y.J. Chen. Small 2 (2006) 485-489.

150. Y.S. Lin, P.J. Tsai, M.F. Weng, Y.C. Chen. Anal. Chem. 77 (2005) 1753-1760.

151. M. Brinkley. Bioconjug Chem. 3 (1992) 2-13.

152. B. Dubertret, P. Skourides, D.J. Norris, V. Noireaux, A.H. Brivanlou, A. Libchaber. Science 298 (2002) 1759-1762.

153. Y.M. Huh, Y.W. Jun, H.T. Song, S. Kim, J.S. Choi, J.H. Lee. J. Am. Chem. Soc. 127 (2005) 12387-12391. 
154. M.E. Gindy, S.X. Ji, T.R. Hoye, A.Z. Panagiotopoulos, R.K. Prud'homme, Biomacromolecules 9 (2008) 2705-2711.

155. M.A. White, J.A. Johnson, J.T. Koberstein, N.J. Turro. J. Am. Chem. Soc. 128 (2006) 11356-11357.

156. T.H. Galow, A.K. Boal, V.M. Rotello. Adv. Mater. 12 (2000) 576-579.

157. G. Han, C.C. You, B.J. Kim, R.S. Turingan, N.S. Forbes, C.T. Martin, V.M. Rotello. Angew. Chem. Int. Ed. 45 (2006) 3165-3169.

158. D. Li, G.P. Li, W.W. Guo, P.C. Li, E.K. Wang, J. Wang. Biomaterials 29 (2008) 2776-2782.

159. N. Nagatani, M. Shinkai, Y. Nagase, H. Honda, K. Hata, H. Mizuno. Biotechnol. Lett. 22 (2000) 999-1002.

160. K.C. Ho, P.J. Tsai, Y.S. Lin, Y.C. Chen. Anal. Chem. 76 (2004) 7162-7168.

161. C.E. Mora-Huertas, H. Fessi, A. Elaissari. Int. J. Pharm. 385 (2010) 113-142.

162. D.E. Owens, N.A. Peppas. Int. J. Pharm. 3 (2006) 93-102. 